\title{
On average Hewitt-Stromberg measures of typical compact metric spaces
}

\section{Olsen ${ }^{1}$}

Received: 11 January 2018 / Accepted: 7 December 2018 / Published online: 24 January 2019

(c) The Author(s) 2019

\section{Abstract}

We study average Hewitt-Stromberg measures of typical compact metric spaces belonging to the Gromov-Hausdorff space (of all compact metric spaces) equipped with the GromovHausdorff metric.

Keywords Box dimension - Compact metric space - The Gromov-Hausdorff metric . Hewitt-Stromberg measures $\cdot$ Hausdorff measure $\cdot$ Packing measure

Mathematics Subject Classification $28 \mathrm{~A} 78 \cdot 28 \mathrm{~A} 80$

\section{Introduction}

Recall that a subset $E$ of a metric space $M$ is called co-meagre if its complement is meagre; also recall that if $\mathrm{P}$ is a property that the elements of $M$ may have, then we say that a typical element $x$ in $M$ has property $\mathrm{P}$ if the set $E=\{x \in M \mid x$ has property $\mathrm{P}\}$ is co-meagre, see Oxtoby [16] for more details. The purpose of this paper is to investigate the average Hewitt-Stromberg measures of a typical compact metric space belonging to the GromovHausdorff space $K_{\mathrm{GH}}$ of all compact metric spaces; the precise definitions of the average Hewitt-Stromberg measures and the Gromov-Hausdorff space $K_{\mathrm{GH}}$ will be given below.

Recall that a dimension function is an increasing and right continuous function $h$ : $(0, \infty) \rightarrow(0, \infty)$ with $h(r) \rightarrow 0$ as $r \rightarrow 0$. The two most important and commonly used fractal measure of a metric space $X$ are the $h$-dimensional Hausdorff measure $\mathcal{H}^{h}(X)$ and the $h$-dimensional packing measure $\mathcal{P}^{h}(X)$ associated with the dimension function $h$; the precise definitions of $\mathcal{H}^{h}(X)$ and $\mathcal{P}^{h}(X)$ will be given in Sect. 2.2. It is well-known that these measures satisfy the following inequality,

$$
\mathcal{H}^{h}(X) \leq \mathcal{P}^{h}(X)
$$

However, there are two further and (perhaps) equally important but less well-known fractal measures of a metric space $X$, namely, the lower and upper Hewitt-Stromberg measures

L. Olsen

lo@st-and.ac.uk

1 Department of Mathematics, University of St. Andrews, St. Andrews, Fife KY16 9SS, Scotland, UK 
associated with the dimension function $h$; the lower and upper Hewitt-Stromberg measures of $X$ associated with the dimension function $h$ will be denoted by $\mathcal{U}^{h}(X)$ and $\mathcal{V}^{h}(X)$, respectively. The Hausdorff measure, the packing measure and the Hewitt-Stromberg measures satisfy the following string of inequalities

$$
\mathcal{H}^{h}(X) \leq \mathcal{U}^{h}(X) \leq \mathcal{V}^{h}(X) \leq \mathcal{P}^{h}(X) ;
$$

in particular, the Hewitt-Stromberg measures form a natural interpolation between the Hausdorff measure and the packing measure. Hewitt-Stromberg measures were introduced by Hewitt and Stromberg in an intriguing exercise in their classical textbook from 1965 [10, (10.51)], and have subsequently been investigated further, see, for example [7,8,22], highlighting their importance in the study of local properties of fractals and products of fractals. In particular, Edgar's textbook [2, pp. 32-36] provides an excellent and systematic introduction to Hewitt-Stromberg measures, and the measures also appear explicitly in, for example, Pesin's monograph [17, 5.3] and implicitly in Mattila's text [13].

We now return to the main question: what are the fractal measures of a typical compact metric space? We are, of course, not the first to ask this question. Indeed, many different aspects of this problem have been studied by several authors during the past 20 years, including $[1,4,6,12]$ and the references therein, and the question also appears implicitly in [5]. While (almost) all previous work, including, for example, [1,4-6], study fractal measures of typical compact subsets of a given complete metric space, this paper adopts a new and different viewpoint introduced very recently by Rouyer [20] and investigated further in [12], namely, we investigate typical compact metric spaces belonging to the Gromov-Hausdorff space $K_{\mathrm{GH}}$ of all compact metric spaces. For example, in [12] the authors prove the following result about the fractal measures of a typical compact metric spaces belonging to the Gromov-Hausdorff space $K_{\mathrm{GH}}$.

Theorem A [12] Let h be a continuous dimension function. A typical compact metric space $X \in K_{G H}$ satisfies

$$
\mathcal{H}^{h}(X)=\mathcal{U}^{h}(X)=0, \quad \mathcal{V}^{h}(X)=\mathcal{P}^{h}(X)=\infty .
$$

We immediately note that the result in Theorem A is qualitatively similar to the behaviour of the Hausdorff measure of a typical compact subset of a fixed complete metric space. Indeed, it follows from [1, Remark 4.3] that if $h$ is a right-continuous dimension function and $X$ is a given fixed complete metric space, then a typical compact subset $K$ of $X$ satisfies $\mathcal{H}^{h}(K)=0$. We also note that Theorem A shows that the lower Hewitt-Stromberg measure (and hence the Hausdorff measure and the Hausdorff dimension) of a typical compact metric space is as small as possible and that the upper Hewitt-Stromberg measure (and hence the packing measure and the packing dimension) of a typical compact metric space is as big as possible. Other studies of typical compact sets, see $[5,15,20]$, show the same dichotomy. For example, [20] proves that a typical compact metric space has lower box dimension equal to 0 and upper box dimension equal to $\infty$, and Gruber [5] and Myjak and Rudnicki [15] prove that if $X$ is a metric space, then the lower box dimension of a typical compact subset of $X$ is as small as possible and that the upper box dimension of a typical compact subset of $X$ is (in many cases) as big as possible. The purpose of this paper is to analyse this intriguing dichotomy, and, in particular, the dichotomy in Theorem A, in more detail. We will prove that the behaviour of a typical compact metric space is spectacularly more irregular than suggested by Theorem $\mathrm{A}$ and the results in $[5,15,20]$. Namely, there are standard techniques, known as averaging systems, that (at least in some cases) can assign limiting values to divergent functions; the precise definition of an averaging system will be given in Sect. 2.5 below. This technique can 
be applied to the definition of the Hewitt-Stromberg measures as follows. Namely, for $E \subseteq X$ and $r>0$, let $M_{r}(E)$ denote the largest number of pairwise disjoint closed balls in $X$ with centres in $E$ and radii equal to $r$, and for a dimension function $h$, define the $h$-dimensional box counting function $F_{E}^{h}(r)$ of $E$ by

$$
F_{E}^{h}(r)=M_{r}(E) h(2 r) .
$$

The Hewitt-Stromberg measures $\mathcal{U}^{h}(E)$ and $\mathcal{V}^{h}(E)$ of a subset $E$ of $X$ are defined in terms of the lower and upper limits of the box counting function $F_{E}^{h}(r)$ of $E$, namely,

$$
\begin{aligned}
& \mathcal{U}^{h}(E)=\inf _{E \subseteq \cup_{i=1}^{\infty} E_{i}} \sum_{i=1}^{\infty} \liminf _{r \searrow 0} F_{E_{i}}^{h}(r), \\
& \mathcal{V}^{h}(E)=\inf _{E \subseteq \cup_{i=1}^{\infty} E_{i}} \sum_{i=1}^{\infty} \limsup _{r \searrow 0} F_{E_{i}}^{h}(r) ;
\end{aligned}
$$

i.e. the measures $\mathcal{U}^{h}$ and $\mathcal{V}^{h}$ are obtained by applying Munroe's Method I to the set functions $E \mapsto \liminf _{r \searrow 0} F_{E}^{h}(r)$ and $E \mapsto \lim _{\sup _{r}{ }^{\prime}} F_{E}^{h}(r)$, see [14]. Fixing an averaging system, $\Pi$ say, and replacing the lower and upper limits of $F_{E_{i}}^{h}(r)$ in (1.2) by the lower and upper $\Pi$ averages of $F_{E_{i}}^{h}(r)$ yields the lower and upper average Hewitt-Stromberg measures $\mathcal{U}_{\Pi}^{h}(X)$ and $\mathcal{V}_{\Pi}^{h}(X)$; see Sect. 2.5 for precise definitions of this. The average Hewitt-Stromberg measures form an interpolation between the Hausdoff measure and the packing measure that is finer than the interpolation (1.1) provided by the Hewitt-Stromberg measures; more precisely, we have

$$
\mathcal{H}^{h}(X) \leq \mathcal{U}^{h}(X) \leq \mathcal{U}_{\Pi}^{h}(X) \leq \mathcal{V}_{\Pi}^{h}(X) \leq \mathcal{V}^{h}(X) \leq \mathcal{P}^{h}(X) .
$$

The purpose of this paper is to show the following (surprising?) result: not only is the box counting function $F_{X}^{h}(r)$ of a typical compact metric space $X$ so divergent that $\mathcal{U}^{h}(X)=0$ and $\mathcal{V}^{h}(X)=\infty$, but it is so irregular that it remains spectacularly divergent even after being "averaged" or "smoothened out" by very general averaging systems $\Pi$ (satisfying the mild closure-stability condition in Sect. 2.6). Specifically, if $\Pi$ is an averaging system, then the associated average Hewitt-Stromberg measures satisfy $\mathcal{U}_{\Pi}^{h}(X)=0$ and $\mathcal{V}_{\Pi}^{h}(X)=\infty$ for a typical compact metric space $X$; more precisely, we prove the following theorem.

Theorem 1.1 (Special case of Theorem 2.5) Let h be a continuous dimension function and let $\Pi$ be an averaging system satisfying the h-closure stability condition in Sect. 2.6. A typical compact metric space $X \in K_{G H}$ satisfies

$$
\mathcal{H}^{h}(X)=\mathcal{U}^{h}(X)=\mathcal{U}_{\Pi}^{h}(X)=0, \quad \mathcal{V}_{\Pi}^{h}(X)=\mathcal{V}^{h}(X)=\mathcal{P}^{h}(X)=\infty .
$$

We present several applications of this result. For example, as an application of Theorem 1.1 we show that a typical compact metric space $X$ is so irregular that the lower (upper) average Hewitt-Stromberg measures associated with all higher order Hölder averages of the box counting function $F_{X}^{h}(r)$ equal $0(\infty)$; below we state a precise version of this and refer the reader to Theorem 3.1 for a more general version of the result.

Theorem 1.2 (Special case of Theorem 3.1) Let $h$ be a continuous dimension function and $n \in \mathbb{N} \cup\{0\}$. We define the $n$ 'th order Hölder averages, denoted by $F_{E, n}^{h}(t)$, of the box counting function $F_{E}^{h}(r)$ of a subset $E$ of a metric space $X$ inductively by

$$
F_{E, 0}^{h}(t)=F_{E}^{h}\left(e^{-t}\right), \quad F_{E, n}^{h}(t)=\frac{1}{t} \int_{1}^{t} F_{E, n-1}^{h}(s) d s,
$$


and we define the lower and upper n'th order Hölder average Hewitt-Stromberg measures of $X$ by

$$
\begin{aligned}
& \mathcal{U}_{n}^{H, h}(X)=\inf _{X=\cup_{i=1}^{\infty} E_{i}} \sum_{i=1}^{\infty} \liminf _{t \rightarrow \infty} F_{E_{i}, n}^{h}(t), \\
& \mathcal{V}_{n}^{H, h}(X)=\inf _{X=\cup_{i=1}^{\infty} E_{i}} \sum_{i=1}^{\infty} \limsup _{t \rightarrow \infty} F_{E_{i}, n}^{h}(t) .
\end{aligned}
$$

The higher order Hölder average Hewitt-Stromberg measures form a double infinite hierarchy between the Hausdorff measure and the packing measure in (at least) countably infinite many levels, namely,

$\mathcal{H}^{h}(X) \leq \mathcal{U}^{h}(X)=\mathcal{U}_{0}^{H, h}(X) \leq \mathcal{U}_{1}^{H, h}(X) \leq \cdots \leq \mathcal{V}_{1}^{H, h}(X) \leq \mathcal{V}_{0}^{H, h}(X)=\mathcal{V}^{h}(X) \leq \mathcal{P}^{h}(X)$, and a typical compact metric space $X \in K_{G H}$ satisfies

$$
\mathcal{U}_{n}^{H, h}(X)=0, \quad \mathcal{V}_{n}^{H, h}(X)=\infty,
$$

for all $n \in \mathbb{N} \cup\{0\}$.

We emphasise that Theorems 1.1 and 1.2 are special cases of more general results presented in Sect. 2.

The paper is structured as follows. We first recall the definitions of the Gromov-Hausdorff space and the Gromov-Hausdorff metric in Sect. 2.1. In Sects. 2.2-2.3 we recall the definitions of the various fractal measures investigated in the paper. The definitions of the Hausdorff and packing measures are recalled in Sect. 2.2 and the definitions of the Hewitt-Stromberg measures are recalled in Sect. 2.3; while the definitions of the Hausdorff and packing measures are well-known, we have, nevertheless, decided to include these - there are two main reasons for this: firstly, to make it easier for the reader to compare and contrast the Hausdorff and packing measurers with the less well-known Hewitt-Stromberg measures, and secondly, to provide a motivation for the Hewitt-Stromberg measures. Section 2.4 recalls earlier results on the values of the Hausdorff measure, the packing measure and the Hewitt-Stromberg measures of typical compact metric spaces; this discussion is included in order to motivate our main results presented Sects. 2.5-2.6. In Sect. 2.5 we define average Hewitt-Stromberg measures, and in Sect. 2.6 we compute the exact values of average Hewitt-Stromberg measures of typical compact metric spaces. In Sect. 3 we apply the main results from Sects. 2.5-2.6 to the detailed study of average Hewitt-Stromberg measures associated with two of the most important types of averages, namely, higher order Hölder averages and higher order Cesaro averages. Finally, the proofs are given in Sects. 4-6.

\section{Statements of results}

\subsection{The Gromov-Hausdorff space $K_{\mathrm{GH}}$ and the Gromov-Hausdorff metric $d_{\mathrm{GH}}$}

We define the pre-Gromov-Hausdorff space $\mathcal{K}_{\mathrm{GH}}$ by

$$
\mathcal{K}_{\mathrm{GH}}=\{X \mid X \text { is a compact and non-empty metric space }\} .
$$

Next, we define the equivalence relation $\sim$ in $\mathcal{K}_{\mathrm{GH}}$ as follows, namely, for $X, Y \in \mathcal{K}_{\mathrm{GH}}$, write

$$
X \sim Y \quad \Leftrightarrow \quad \text { there is a bijective isometry } f: X \rightarrow Y \text {. }
$$


It is clear that $\sim$ is an equivalence relation in $\mathcal{K}_{\mathrm{GH}}$, and the Gromov-Hausdorff space $K_{\mathrm{GH}}$ is now defined as the space of equivalence classes, i.e.

$$
K_{\mathrm{GH}}=\mathcal{K}_{\mathrm{GH}} / \sim \text {. }
$$

While elements of $K_{\mathrm{GH}}$ are equivalence classes of compact metric spaces, we will use the standard convention and identify an equivalence class with it representative, i.e. we will regard the elements of $K_{\mathrm{GH}}$ as compact metric spaces and not as equivalence classes of compact metric spaces. Next, we define the Gromov-Hausdorff metric $d_{\mathrm{GH}}$ on $K_{\mathrm{GH}}$. If $Z$ is a metric space, and $A$ and $B$ are compact subsets of $Z$, then the Hausdorff distance $d_{\mathrm{H}}(A, B)$ between $A$ and $B$ is defined by

$$
d_{\mathrm{H}}(A, B)=\max \left(\sup _{x \in A} \operatorname{dist}(x, B), \sup _{y \in B} \operatorname{dist}(y, A)\right),
$$

where $\operatorname{dist}(z, E)=\inf _{x \in E} d(z, x)$ for $z \in Z$ and $E \subseteq Z$. The Gromov-Hausdorff metric $d_{\mathrm{GH}}$ on $K_{\mathrm{GH}}$ is now define by

$$
\begin{aligned}
d_{\mathrm{GH}}(X, Y)= & \inf \left\{d_{\mathrm{H}}(f(X), g(Y)) \mid Z\right. \text { is a complete metric space } \\
& \text { and } f: X \rightarrow Z \text { and } g: Y \rightarrow Z \text { are isometries }\} .
\end{aligned}
$$

for $X, Y \in K_{\mathrm{GH}}$. The reader is referred to [18, Chapter 10] for a detailed discussion of the Gromov-Hausdorff space and the Gromov-Hausdorff metric. In particular, we note that it follows from [18] that the Gromov-Hausdorff space $\left(K_{\mathrm{GH}}, d_{\mathrm{GH}}\right)$ is complete (and, hence, not meagre) and the classification of subsets of $K_{\mathrm{GH}}$ using Baire category is therefore meaningful.

\subsection{Hausdorff measure and packing measure}

While the definitions of the Hausdorff and packing measures (and the Hausdorff and packing dimensions) are well-known, we have, nevertheless, decided to briefly recall the definitions below. There are several reasons for this: firstly, since we are working in general (compact) metric spaces, the different definitions that appear in the literature may not all agree and for this reason it is useful to state precisely the definitions that we are using; secondly, and perhaps more importantly, the less well-known Hewitt-Stromberg measures (which will be defined below in Sect. 2.3) play an important part in this paper and to make it easier for the reader to compare and contrast the definitions of the Hewitt-Stromberg measures and the definitions of the Hausdorff and packing measures it is useful to recall the definitions of the latter measures; thirdly, in order to provide a motivation for the Hewitt-Stromberg measures. We start by recalling the definition of a dimension function.

Definition (Dimension function) A function $h:(0, \infty) \rightarrow(0, \infty)$ is called a dimension function if $h$ is increasing, right continuous and $\lim _{r \searrow_{0}} h(r)=0$.

The Hausdorff measure associated with a dimension function $h$ is defined as follows. Let $X$ be a metric space and $E \subseteq X$. For $\delta>0$, we write

$$
\mathcal{H}_{\delta}^{h}(E)=\inf \left\{\sum_{i} h\left(\operatorname{diam}\left(E_{i}\right)\right) \mid E \subseteq \bigcup_{i=1}^{\infty} E_{i}, \operatorname{diam}\left(E_{i}\right)<\delta\right\} .
$$

The $h$-dimensional Hausdorff measure $\mathcal{H}^{h}(E)$ of $E$ is now defined by

$$
\mathcal{H}^{h}(E)=\sup _{\delta>0} \mathcal{H}_{\delta}^{h}(E) .
$$


The reader is referred to Rogers classical text for [19] for an excellent and systematic discussion the Hausdorff measures $\mathcal{H}^{h}$.

The packing measure associated with a dimension function $h$ is defined as follows. For $x \in X$ and $r>0$, let $C(x, r)$ denote the closed ball in $X$ with centre at $x$ and radius equal to $r$, and for $\delta>0$, write

$$
\begin{aligned}
& \overline{\mathcal{P}}_{\delta}^{h}(E)=\sup \left\{\sum_{i=1}^{\infty} h\left(2 r_{i}\right) \mid\left(C\left(x_{i}, r_{i}\right)\right)_{i=1}^{\infty} \text { is a family of pairwise disjoint closed balls in } X\right. \\
& \text { with } \left.r_{i}<\delta \text { and centres in } E\right\} .
\end{aligned}
$$

The $h$-dimensional prepacking measure $\overline{\mathcal{P}}^{h}(E)$ of $E$ is now defined by

$$
\overline{\mathcal{P}}^{h}(E)=\inf _{\delta>0} \overline{\mathcal{P}}_{\delta}^{h}(E)
$$

and the $h$-dimensional packing measure $\mathcal{P}^{t}(E)$ of $E$ is defined as follows

$$
\mathcal{P}^{h}(E)=\inf _{E \subseteq \cup_{i=1}^{\infty} E_{i}} \sum_{i=1}^{\infty} \overline{\mathcal{P}}^{h}\left(E_{i}\right) .
$$

\subsection{Hewitt-Stromberg measures}

Hewitt-Stromberg measures were introduced by Hewitt and Stromberg in their classical textbook [10, (10.51)]. While Hausdorff and packing measures are defined using coverings and packings by families of sets with diameters less than a given positive number, $\delta$ say, the Hewitt-Stromberg measures are defined using packings of balls with the same diameter $\delta$.

We will now recall the definition the Hewitt-Stromberg measures. Let $X$ be a metric space and $E \subseteq X$. We first recall the definition of the packing number of $E$. For $r>0$, the packing number $M_{r}(E)$ of $E$ is defined by

$$
\begin{gathered}
M_{r}(X)=\sup \{|\mathcal{B}| \mid \mathcal{B} \text { is a family of pairwise disjoint closed balls in } X \\
\quad \text { with radii equal to } r \text { and centres in } E\} .
\end{gathered}
$$

Next, we recall the definition of the Hewitt-Stromberg measures associated with a dimension function $h$. For a metric space $X$ and $E \subseteq X$, we define the lower and upper $h$-dimensional Hewitt-Stromberg pre-measures, denote by $\overline{\mathcal{U}}^{h}$ and $\overline{\mathcal{V}}^{h}$, respectively, by

$$
\begin{aligned}
& \overline{\mathcal{U}}^{h}(E)=\liminf _{r \searrow 0} M_{r}(E) h(2 r) . \\
& \overline{\mathcal{V}}^{h}(E)=\limsup _{r \searrow 0} M_{r}(E) h(2 r) .
\end{aligned}
$$


Finally, we define the lower and upper $h$-dimensional Hewitt-Stromberg measures, denote by $\mathcal{U}^{h}$ and $\mathcal{V}^{h}$, respectively, by

$$
\begin{aligned}
& \mathcal{U}^{h}(E)=\inf _{E \subseteq \cup_{i=1}^{\infty} E_{i}} \sum_{i=1}^{\infty} \overline{\mathcal{U}}^{h}\left(E_{i}\right), \\
& \mathcal{V}^{h}(E)=\inf _{E \subseteq \cup_{i=1}^{\infty} E_{i}} \sum_{i=1}^{\infty} \overline{\mathcal{V}}^{h}\left(E_{i}\right) .
\end{aligned}
$$

The next result summarises the basic inequalities satisfied by the Hewitt-Stromberg measures, the Hausdorff measure and the packing measure.

Proposition 2.1 Let $h$ be a dimension function. Then we have

$$
\begin{array}{rl}
\overline{\mathcal{U}}^{h}(E) & \leq \overline{\mathcal{V}}^{h}(E) \leq \overline{\mathcal{P}}^{h}(E) \\
\mathrm{VI} & \mathrm{VI} \\
\mathcal{H}^{h}(E) \leq \mathcal{U}^{h}(E) & \leq \mathcal{V}^{h}(E) \leq \mathcal{P}^{h}(E)
\end{array}
$$

for all metric spaces $X$ and all $E \subseteq X$.

Proof This follows immediately from the definitions; see also [2, pp. 32-36].

\subsection{Hausdorff measures, packing measures and Hewitt-Stromberg measures of typical compact spaces}

Jurina et al [12] have recently computed the Hausdorff measures, the packing measures and the Hewitt-Stromberg measures of typical compact spaces; this is the content of Theorem B below.

Theorem B [12] Hausdorff measures, packing measures and Hewitt-Stromberg measures of typical compact spaces. Let $h$ be a continuous dimension function.

(1) A typical compact metric space $X \in K_{G H}$ satisfies

$$
\mathcal{H}^{h}(X)=\mathcal{U}^{h}(X)=\overline{\mathcal{U}}^{h}(X)=0 .
$$

(2) A typical compact metric space $X \in K_{G H}$ satisfies

$$
\mathcal{V}^{h}(U)=\overline{\mathcal{V}}^{h}(U)=\mathcal{P}^{h}(U)=\infty
$$

for all non-empty open subsets $U$ of $X$. In particular, a typical compact metric space $X \in K_{G H}$ satisfies

$$
\mathcal{V}^{h}(X)=\overline{\mathcal{V}}^{h}(X)=\mathcal{P}^{h}(X)=\infty
$$

Theorem B shows that the lower Hewitt-Stromberg pre-measure and the lower HewittStromberg measure of a typical compact metric space are as small as possible and that the upper Hewitt-Stromberg pre-measure and the upper Hewitt-Stromberg measure of a typical compact metric space are as big as possible. We will analyse this intriguing dichotomy in more details by forming different types of averages. In order to do so, we introduce the following notation. Namely, for a dimensions function $h$ and a subset $E$ of a metric space $X$, we define the $h$-dimensional box counting function $f_{E}^{h}:(0, \infty) \rightarrow[0, \infty]$ of $E$ by

$$
f_{E}^{h}(t)=M_{e^{-t}}(E) h\left(2 e^{-t}\right)
$$


(hence, the $h$-dimensional box counting function $f_{E}^{h}(t)=M_{e^{-t}}(E) h\left(2 e^{-t}\right)$ in (2.2) is obtained from the $h$-dimensional box counting function $F_{E}^{h}(r)=M_{r}(E) h(2 r)$ in Sect. 1 by introducing the following change of variables, namely, by letting $r=e^{-t}$; the reason for this change of variables is that it is more convenient to let $t \rightarrow \infty$ when forming averages than letting $r \searrow 0$ ). Using the notation from (2.1), the Hewitt-Stromberg pre-measures of $X$ are now given by

$$
\begin{aligned}
& \overline{\mathcal{U}}^{h}(X)=\liminf _{t \rightarrow \infty} f_{X}^{h}(t), \\
& \overline{\mathcal{V}}^{h}(X)=\limsup _{t \rightarrow \infty} f_{X}^{h}(t),
\end{aligned}
$$

and Theorem B therefore shows that the $h$-dimensional box counting function $f_{X}^{h}(t)$ of a typical compact metric space $X \in K_{\mathrm{GH}}$ diverges in the worst possible way as $t \rightarrow \infty$. Below we analyse this divergence in detail using average procedures known as average systems.

\subsection{Average Hewitt-Stromberg measures}

We start by recalling the definition of an averaging (or summability) system; the reader is referred to Hardy's classical text [9] for a systematic treatment of averaging systems.

Definition (Average system) An averaging system is a family $\Pi=\left(\Pi_{t}\right)_{t \geq t_{0}}$ with $t_{0}>0$ such that:

(i) $\Pi_{t}$ is a finite Borel measure on $\left[t_{0}, \infty\right)$;

(ii) $\Pi_{t}$ has compact support;

(iii) The Consistency Condition: If $f:\left[t_{0}, \infty\right) \rightarrow[0, \infty)$ is a positive measurable function and there is a real number $a$ such that $f(t) \rightarrow a$ as $t \rightarrow \infty$, then $\int f d \Pi_{t} \rightarrow a$ as $t \rightarrow$ $\infty$.

If $f:\left[t_{0}, \infty\right) \rightarrow[0, \infty)$ is a positive measurable function, then we define lower and upper $\Pi$-average of $f$ by

$$
\underline{A}_{\Pi} f=\liminf _{t \rightarrow \infty} \int f d \Pi_{t}
$$

and

$$
\bar{A}_{\Pi} f=\limsup _{t \rightarrow \infty} \int f d \Pi_{t},
$$

respectively.

Applying averaging systems to the box counting function $f_{E}^{h}(t)$ in (2.2) leads to the definition of average Hewitt-Stromberg measures.

Definition (Average Hewitt-Stromberg measures) Let $h$ be a dimension function and let $\Pi=\left(\Pi_{t}\right)_{t \geq t_{0}}$ be an averaging system. For a metric space $X$ and $E \subseteq X$, we define the lower and upper $\Pi$-average $h$-dimensional Hewitt-Stromberg pre-measures of $E$ by

$$
\overline{\mathcal{U}}_{\Pi}^{h}(E)=\underline{A}_{\Pi} f_{E}^{h}=\liminf _{t \rightarrow \infty} \int f_{E}^{h} d \Pi_{t}
$$

and

$$
\overline{\mathcal{V}}_{\Pi}^{h}(E)=\bar{A}_{\Pi} f_{E}^{h}=\limsup _{t \rightarrow \infty} \int f_{E}^{h} d \Pi_{t}
$$


We define the lower and upper $\Pi$-average $h$-dimensional Hewitt-Stromberg measures of $E$ by

$$
\mathcal{U}_{\Pi}^{h}(E)=\inf _{E \subseteq \cup_{i=1}^{\infty} E_{i}} \sum_{i=1}^{\infty} \overline{\mathcal{U}}_{\Pi}^{h}\left(E_{i}\right)
$$

and

$$
\mathcal{V}_{\Pi}^{h}(E)=\inf _{E \subseteq \cup_{i=1}^{\infty} E_{i}} \sum_{i=1}^{\infty} \overline{\mathcal{V}}_{\Pi}^{h}\left(E_{i}\right),
$$

respectively

Note that Hewitt-Stromberg measures are, in fact, average Hewitt-Stromberg measures. Indeed, if $X$ a metric space and we let $\Pi$ denote the average system defined by $\Pi=\left(\delta_{t}\right)_{t \geq 1}$ (where $\delta_{t}$ denotes the Dirac measure concentrated at $t$ ), then clearly

$$
\mathcal{U}^{h}(E)=\mathcal{U}_{\Pi}^{h}(E), \quad \mathcal{V}^{h}(E)=\mathcal{V}_{\Pi}^{h}(E)
$$

for all subsets $E$ of $X$. Below we list the basic inequalities satisfied by the average HewittStromberg measures, the Hausdorff measure and the packing measure.

Proposition 2.2 Let $h$ be a dimension function and let $\Pi$ be an averaging system. Then we have

$$
\begin{aligned}
& \overline{\mathcal{U}}^{h}(E) \leq \overline{\mathcal{U}}_{\Pi}^{h}(E) \leq \overline{\mathcal{V}}_{\Pi}^{h}(E) \leq \overline{\mathcal{V}}^{h}(E) \leq \overline{\mathcal{P}}^{h}(E) \\
& \begin{array}{lllll}
V I & V I & V I & V I & V I
\end{array} \\
& \mathcal{H}^{h}(E) \leq \mathcal{U}^{h}(E) \leq \mathcal{U}_{\Pi}^{h}(E) \leq \mathcal{V}_{\Pi}^{h}(E) \leq \mathcal{V}^{h}(E) \leq \mathcal{P}^{h}(E)
\end{aligned}
$$

for all metric spaces $X$ and all $E \subseteq X$.

Proof The statement is not difficult to prove and we have therefore decided to omit the proof.

\subsection{Average Hewitt-Stromberg measures of typical compact spaces}

In this section we present our main results. Many of our main results are valid for arbitrary averaging systems. However, in order to obtain the most optimal results we occasionally will have to assume that the averaging system satisfies a mild technical condition, namely, the $h$-closure-stability condition given in the following definition.

Definition ( $h$-closure-stable) Let $h$ be a dimension function. An averaging system $\Pi$ is called $h$-closure-stable if $\overline{\mathcal{U}}_{\Pi}^{h}(\bar{E})=\overline{\mathcal{U}}_{\Pi}^{h}(E)$ and $\overline{\mathcal{V}}_{\Pi}^{h}(\bar{E})=\overline{\mathcal{V}}_{\Pi}^{h}(E)$ for all metric spaces $X$ and all $E \subseteq X$.

We immediately note that two important classes of averaging system are closure-stable. Namely, Proposition 2.3 (below) shows that the trivial averaging system $\Pi=\left(\delta_{t}\right)_{t \geq 1}$ is $h$-closure-stable for any dimension function, and Proposition 2.4 (below) shows that if $\Pi=$ $\left(\Pi_{t}\right)_{t \geq t_{0}}$ is an averaging system and each measure $\Pi_{t}$ has a continuous density with respect to Lebesgue measure, then $\Pi$ is $h$-closure-stable for any continuous dimension function.

Proposition 2.3 The averaging system $\Pi=\left(\delta_{t}\right)_{t \geq 1}$ (where $\delta_{t}$ denotes the Dirac measure concentrated at $t$ ) is $h$-closure-stable for any dimension function $h$. 
Proof This follows easily from the definitions and we have therefore decided to omit the proof.

Proposition 2.4 Let $\Pi=\left(\Pi_{t}\right)_{t \geq t_{0}}$ be an averaging system and assume that for each there is a measurable function $\pi_{t}:\left[t_{0}, \infty\right) \rightarrow[0, \infty)$ satisfying

$$
\Pi_{t}(B)=\int_{B} \pi_{t}(s) d s
$$

for all Borel sets $B$ and such that if we write $T_{t}=\sup \operatorname{supp} \pi_{t}$, then

(i) $\operatorname{supp} \pi_{t}=\left[t_{0}, T_{t}\right]$;

(ii) $\pi_{t}$ is continuous on $\left[t_{0}, T_{t}\right]$;

(iii) $\pi_{t}>0$ on $\left[t_{0}, T_{t}\right]$.

Then $\Pi$ is $h$-closure-stable for any continuous dimension function $h$.

The proof of Proposition 2.4 is given in Sect. 4. We can now state the main result in this paper, namely, Theorem 2.5 below. Theorem 2.5 provides the following (surprising?) extension of Theorem B: not only is the box counting function $f_{X}^{h}(t)=M_{e^{-t}}(X) h\left(2 e^{-t}\right)$ of a typical compact metric space $X$ divergent as $t \rightarrow \infty$, but it is so irregular that it remains spectacularly divergent as $t \rightarrow \infty$ even after being "averaged" or "smoothened out" using very general averaging systems including, as will be shown in Sect. 3, all higher order Hölder and Cesaro averages.

Theorem 2.5 (Average Hewitt-Stromberg measures of typical compact spaces) Let $h$ be a continuous dimension function and let $\Pi$ be an averaging system.

(1) A typical compact metric space $X \in K_{G H}$ satisfies

$$
\overline{\mathcal{U}}_{\Pi}^{h}(X)=0 .
$$

(2) A typical compact metric space $X \in K_{G H}$ satisfies

$$
\overline{\mathcal{V}}_{\Pi}^{h}(U)=\infty
$$

for all non-empty open subsets $U$ of $X$.

(3) If, in addition, $\Pi$ is h-closure-stable, then a typical compact metric space $X \in K_{G H}$ satisfies

$$
\mathcal{V}_{\Pi}^{h}(U)=\infty
$$

for all non-empty open subsets $U$ of $X$.

The proof of Theorem 2.5 is given in Sects. 4-6. Section 4 contains various preliminary auxiliary results; the proof of Theorem 2.5.(1) is given in Sect. 5, and the proofs of Theorem 2.5.(2)-(3) are given in Sect. 6.

Note that if we apply Theorem 2.5 to the trivial average system $\Pi$ defined by $\Pi=\left(\delta_{t}\right)_{t \geq 1}$, then it follows from (2.4) that the statement in Theorem 2.5 reduces to Theorem B.

We will now apply Theorem 2.5 to study average Hewitt-Stromberg measures obtained by considering higher order Hölder and Cesaro averages of the box counting function $f_{X}^{h}(t)=$ $M_{e^{-t}}(X) h\left(2 e^{-t}\right)$; this is the contents of Sect. 3 below. 


\section{Hölder and Cesaro averages of Hewitt-Strimborg measurers of a typical compact metric space}

Two of the most commonly used averaging system are Hölder averages and Cesaro averages. We will now define these average systems and apply them to the box counting function $f_{X}^{h}(t)=M_{e^{-t}}(X) h\left(2 e^{-t}\right)$ of a metric space $X$. For $a>0$ and a positive measurable function $f:(a, \infty) \rightarrow[0, \infty)$, we define $M f:(a, \infty) \rightarrow[0, \infty)$ by

$$
(M f)(t)=\frac{1}{t} \int_{a}^{t} f(s) d s .
$$

For a positive integer $n$, we now define the lower and upper $n$ 'th order Hölder averages of $f$ by

$$
\begin{aligned}
& \underline{H}_{n} f=\liminf _{t \rightarrow \infty}\left(M^{n} f\right)(t), \\
& \bar{H}_{n} f=\limsup _{t \rightarrow \infty}\left(M^{n} f\right)(t) .
\end{aligned}
$$

The Cesaro averages are defined as follows. First, we define If $:(a, \infty) \rightarrow[0, \infty)$ by

$$
(I f)(t)=\int_{a}^{t} f(s) d s .
$$

For a positive integer $n$, we now define the lower and upper $n$ 'th order Cesaro averages of $f$ by

$$
\begin{aligned}
& \underline{C}_{n} f=\liminf _{t \rightarrow \infty} \frac{n !}{t^{n}}\left(I^{n} f\right)(t), \\
& \bar{C}_{n} f=\limsup _{t \rightarrow \infty} \frac{n !}{t^{n}}\left(I^{n} f\right)(t) .
\end{aligned}
$$

It is well-known that the Hölder and Cesaro averages satisfy the following inequalities, namely,

$$
\begin{aligned}
& \liminf _{t \rightarrow \infty} f(t)=\underline{H}_{0} f \leq \underline{H}_{1} f \leq \underline{H}_{2} f \leq \cdots \leq \bar{H}_{2} f \leq \bar{H}_{1} f \leq \bar{H}_{0} f=\limsup _{t \rightarrow \infty} f(t), \\
& \liminf _{t \rightarrow \infty} f(t)=\underline{C}_{0} f \leq \underline{C}_{1} f \leq \underline{C}_{2} f \leq \cdots \leq \bar{C}_{2} f \leq \bar{C}_{1} f \leq \bar{C}_{0} f=\limsup _{t \rightarrow \infty} f(t) .
\end{aligned}
$$

It is also well-known that the Hölder and Cesaro averages are averaging systems in the sense of the definition in Sect. 2.5. Indeed, if we for a positive integer $n$, define the averaging system $\Pi_{n}^{\mathrm{H}}=\left(\Pi_{n, t}^{\mathrm{H}}\right)_{t \geq a}$ by

$$
\Pi_{n, t}^{\mathrm{H}}(B)=\frac{1}{(n-1) ! t} \int_{[a, t] \cap B}(\log t-\log s)^{n-1} d s
$$

for Borel subsets $B$ of $[a, \infty)$, then

$$
\begin{aligned}
& \underline{H}_{n} f=\liminf _{t} \int f d \Pi_{n, t}^{\mathrm{H}}, \\
& \bar{H}_{n} f=\limsup _{t} \int f d \Pi_{n, t}^{\mathrm{H}},
\end{aligned}
$$

see, for example, [11, p. 675]. Similarly, if we for a positive integer $n$, define the averaging system $\Pi_{n}^{\mathrm{C}}=\left(\Pi_{n, t}^{\mathrm{C}}\right)_{t \geq a}$ by 


$$
\Pi_{n, t}^{\mathrm{C}}(B)=\frac{n}{t^{n}} \int_{[a, t] \cap B}(t-s)^{n-1} d s
$$

then

$$
\begin{aligned}
& \underline{C}_{n} f=\liminf _{t} \int f d \Pi_{n, t}^{c}, \\
& \bar{C}_{n} f=\limsup _{t} \int f d \Pi_{n, t}^{C},
\end{aligned}
$$

see, for example, [9, pp. 110-111].

Using Hölder and Cesaro averages we can now introduce average Hölder and Cesaro Hewitt-Stromberg measures by applying the definitions of the Hölder and Cesaro averages to the function $f_{X}^{h}(t)=M_{e^{-t}}(X) h\left(2 e^{-t}\right)$. This is the content of the next definition.

Definition (Average Hölder and Cesaro Hewitt-Stromberg measures) Let $X$ be a metric space and $n$ an integer with $n \geq 0$. We define the lower and upper $n$ 'th order average Hölder Hewitt-Stromberg measures of a subset $E$ of $X$, denoted $\mathcal{U}_{n}^{\mathrm{H}, h}(E)$ and $\mathcal{V}_{n}^{\mathrm{H}, h}(E)$, by

$$
\begin{aligned}
& \mathcal{U}_{n}^{\mathrm{H}, h}(E)=\mathcal{U}_{\Pi_{n}^{\mathrm{H}}}^{h}(E), \\
& \mathcal{V}_{n}^{\mathrm{H}, h}(E)=\mathcal{V}_{\Pi_{n}^{\mathrm{H}}}^{h}(E) .
\end{aligned}
$$

Similarly, we define the lower and upper $n$ 'th order average Cesaro Hewitt-Stromberg measures of a subset $E$ of $X$, denoted $\mathcal{U}_{n}^{C, h}(E)$ and $\mathcal{V}_{n}^{\complement, h}(E)$, by

$$
\begin{aligned}
& \mathcal{U}_{n}^{C, h}(E)=\mathcal{U}_{\Pi_{n}^{C}}^{h}(E), \\
& \mathcal{V}_{n}^{C, h}(E)=\mathcal{V}_{\Pi_{n}^{C}}^{h}(E) .
\end{aligned}
$$

The higher order average Hölder and Cesaro Hewitt-Stromberg measures form a double infinite hierarchy between the Hausdorff measure and the packing measure in (at least) countably infinite many levels, namely, we have (using (3.1))

$$
\begin{aligned}
& \mathcal{H}^{h}(E) \leq \mathcal{U}^{h}(E)=\mathcal{U}_{0}^{\mathrm{H}, h}(E) \leq \mathcal{U}_{1}^{\mathrm{H}, h}(E) \leq \cdots \leq \mathcal{V}_{1}^{\mathrm{H}, h}(E) \leq \mathcal{V}_{0}^{\mathrm{H}, h}(E)=\mathcal{V}^{h}(E) \leq \mathcal{P}^{h}(E), \\
& \mathcal{H}^{h}(E) \leq \mathcal{U}^{h}(E)=\mathcal{U}_{0}^{\complement, h}(E) \leq \mathcal{U}_{1}^{\complement, h}(E) \leq \cdots \leq \mathcal{V}_{1}^{\complement, h}(E) \leq \mathcal{V}_{0}^{\complement, h}(E)=\mathcal{V}^{h}(E) \leq \mathcal{P}^{h}(E)
\end{aligned}
$$

As an application of Theorem 2.5, we will now show that the behaviour of a typical compact metric space $X \in K_{\mathrm{GH}}$ is so irregular that not even the hierarchies in (3.2) formed by taking Hölder and Cesaro averages of all orders are sufficiently powerful to "smoothen out" the behaviour of the box counting function $f_{X}^{h}(t)=M_{e^{-t}}(X) h\left(2 e^{-t}\right)$ as $t \rightarrow \infty$.

Theorem 3.1 Let $h$ be a continuous dimension function. A typical compact metric space $X \in K_{G H}$ satisfies

$$
\mathcal{U}_{n}^{H, h}(X)=\mathcal{U}_{n}^{C, h}(X)=0
$$

for all $n \in \mathbb{N} \cup\{0\}$, and

$$
\mathcal{V}_{n}^{H, h}(U)=\mathcal{V}_{n}^{C, h}(U)=\infty
$$

for all non-empty open subsets $U$ of $X$ and all $n \in \mathbb{N} \cup\{0\}$.

Proof This statement follows immediately from Theorem 2.5. 


\section{Proof of Proposition 2.4}

We now turn towards the proof of Proposition 2.4. More precisely, the purpose of this section is threefold. Firstly, we recall a technical auxiliary result due to Gruber [5] and Rouyer [20] about the packing number (defined in (2.1)) and the covering number (defined below) of a metric space; this result plays an important role in Sects. 5, 6 and is stated in Lemma 4.1. Secondly, we prove an auxiliary results about the average $h$-dimensional Hewitt-Stromberg measures associated with $h$-closure-stable average systems; this result also plays an important role in Sects. 5, 6 and is proven in Lemma 4.2. Thirdly, and finally, we prove Proposition 2.4 showing that if $\Pi=\left(\Pi_{t}\right)_{t \geq t_{0}}$ is an averaging system and each measure $\Pi_{t}$ has a continuous density with respect to Lebesgue measure, then $\Pi$ is $h$-closure-stable for any continuous dimension function $h$.

We first recall the statement in Lemma 4.1 below; this is a well-known result due to Gruber [5] and Rouyer [20] and lists some useful continuity properties of the packing number and the covering number of a metric space. Recall, that if $X$ is a metric space and $E \subseteq X$, then the packing number $M_{r}(E)$ of $E$ is defined by

$$
\begin{aligned}
M_{r}(X)= & \sup \{|\mathcal{B}| \mid \mathcal{B} \text { is a family of pairwise disjoint closed balls in } X \\
& \text { with radii equal to } r \text { and centres in } E\} .
\end{aligned}
$$

We also define the covering number $N_{r}(E)$ of $E$ by

$$
N_{r}(E)=\inf \{|\mathcal{B}| \mid \mathcal{B} \text { is a family of closed balls in } X \text { with radii equal to } r \text { that covers } E\} \text {. }
$$

We can now state Lemma 4.1

Lemma 4.1 (1) The function $N_{r}: K_{G H} \rightarrow \mathbb{R}$ is lower semi-continuous for all $r>0$.

(2) The function $M_{r}: K_{G H} \rightarrow \mathbb{R}$ is upper semi-continuous for all $r>0$.

(3) We have $M_{r}(X) \leq N_{r}(X) \leq M_{\frac{r}{3}}(X)$ for all $r>0$ and all $X \in K_{G H}$.

Proof This follows from [20, Lemma 9]; see also [5].

Secondly, we prove Lemma 4.2 providing a useful technique for establishing lower bounds for the average $h$-dimensional Hewitt-Stromberg measures associated with $h$-closure-stable average systems. Recall (see Sect. 2.6), that if $h$ is a dimension function, then an average system $\Pi$ is called $h$-closure-stable if $\overline{\mathcal{U}}_{\Pi}^{h}(\bar{E})=\overline{\mathcal{U}}_{\Pi}^{h}(E)$ and $\overline{\mathcal{V}}_{\Pi}^{h}(\bar{E})=\overline{\mathcal{V}}_{\Pi}^{h}(E)$ for all metric spaces $X$ and all $E \subseteq X$. We can now state Lemma 4.2.

Lemma 4.2 Let $h$ be a continuous dimension function and let $\Pi=\left(\Pi_{t}\right)_{t \geq t_{0}}$ be an h-closurestable averaging system. Let $X$ be a complete metric space and let $C$ be a compact subset of $X$. Fix $c \geq 0$. Then the following statements hold.

(1) If $\overline{\mathcal{U}}_{\Pi}^{h}(V \cap C) \geq c$ for all open subsets $V$ of $X$ with $V \cap C \neq \varnothing$, then $\mathcal{U}_{\Pi}^{h}(C) \geq c$.

(2) If $\overline{\mathcal{V}}_{\Pi}^{h}(V \cap C) \geq c$ for all open subsets $V$ of $X$ with $V \cap C \neq \varnothing$, then $\mathcal{V}_{\Pi}^{h}(C) \geq c$.

Proof (1) Assume that $\overline{\mathcal{U}}_{\Pi}^{h}(V \cap C) \geq c$ for all open subsets $V$ of $X$ with $V \cap C \neq \varnothing$. We must now show that $\mathcal{U}_{\Pi}^{h}(C) \geq c$. Let $\left(E_{i}\right)_{i}$ be a countable family of subsets of $X$ with $C \subseteq \cup_{i} E_{i}$. Since $C=\cup_{i} E_{i} \subseteq \cup_{i} \overline{E_{i}}$, it follows from Baire's category theorem that there is an index $i_{0}$ 
and an open subset $W$ of $X$ such that $C \cap W \neq \varnothing$ and $C \cap W \subseteq \overline{E_{i_{0}}}$. We therefore conclude that $\overline{\mathcal{U}}_{\Pi}^{h}\left(\overline{E_{i_{0}}}\right) \geq \overline{\mathcal{U}}_{\Pi}^{h}(C \cap W) \geq c$. Since $\Pi$ is $h$-closure-stable, it follows from this that

$$
\sum_{i} \overline{\mathcal{U}}_{\Pi}^{h}\left(E_{i}\right) \geq \overline{\mathcal{U}}_{\Pi}^{h}\left(E_{i_{0}}\right)=\overline{\mathcal{U}}_{\Pi}^{h}\left(\overline{E_{i_{0}}}\right) \geq c .
$$

Finally, using (4.3) and taking infimum over all countable families $\left(E_{i}\right)_{i}$ of subsets of $X$ with $C \subseteq \cup_{i} E_{i}$, shows that $\mathcal{U}_{\Pi}^{h}(E)=\inf _{E \subseteq \cup_{i=1}^{\infty} E_{i}} \sum_{i=1}^{\infty} \overline{\mathcal{U}}_{\Pi}^{h}\left(E_{i}\right) \geq c$.

(2) The proof of this statement is identical to the proof of the statement in Part (1) and is therefore omitted.

Thirdly, and finally, we prove Proposition 2.4 from Sect. 2. We start with a small lemma.

Lemma 4.3 Let $r>0$ and $0<u<1$. Then

$$
M_{r}(\bar{E}) \leq M_{u r}(E) .
$$

for all compact metric spaces $X$ and all $E \subseteq X$.

Proof Write $\delta=1-u \in(0,1)$. It follows from the definition of the packing number $M_{r}(\bar{E})$ that we can find a family $\left(C\left(x_{i}, r\right)\right)_{i=1}^{M_{r}(\bar{E})}$ of pairwise disjoint closed balls $C\left(x_{i}, r\right)$ in $X$ with radii equal to $r$ and centres $x_{i} \in \bar{E}$. Since $x_{i} \in \bar{E}$, there is a point $y_{i} \in E$ such that $y_{i} \in B\left(x_{i}, \delta r\right)$, whence $C\left(y_{i},(1-\delta) r\right) \subseteq C\left(x_{i}, r\right)$. As the family $\left(C\left(x_{i}, r\right)\right)_{i=1}^{M_{r}(\bar{E})}$ consists of pairwise disjoint balls, we therefore conclude that $\left(C\left(y_{i},(1-\delta) r\right)\right)_{i=1}^{M_{r}(\bar{E})}$ is a family of pairwise disjoint closed balls in $X$ with radii equal to $(1-\delta) r$ and centres $y_{i} \in E$. This clearly implies that $M_{r}(\bar{E}) \leq M_{(1-\delta) r}(E)=M_{u r}(E)$.

We can now prove Proposition 2.4.

Proof of Proposition 2.4 Let $X$ be a metric space and $E \subseteq X$. It is clear that $\overline{\mathcal{U}}_{\Pi}^{h}(E) \leq \overline{\mathcal{U}}_{\Pi}^{h}(\bar{E})$ and $\overline{\mathcal{V}}_{\Pi}^{h}(E) \leq \overline{\mathcal{V}}_{\Pi}^{h}(\bar{E})$, and it therefore suffices to prove that $\overline{\mathcal{U}}_{\Pi}^{h}(\bar{E}) \leq \overline{\mathcal{U}}_{\Pi}^{h}(E)$ and $\overline{\mathcal{V}}_{\Pi}^{h}(\bar{E}) \leq$ $\overline{\mathcal{V}}_{\Pi}^{h}(E)$. We will now prove these inequalities. Let $\varepsilon>0$. Fix $t \geq t_{0}$. Next we define three numbers $\rho_{t}, \delta_{t}$ and $u_{t}$ as follows.

Definition of $\rho_{t}$. Write $K_{t}=\left\{(u, v) \mid u \geq t_{0}, v \geq 0, t_{0} \leq u-v \leq T_{t}\right\}$ and define $D_{t}: K_{t} \rightarrow[0, \infty)$ by $D_{t}(u, v)=\frac{\pi_{t}(u-v)}{\pi_{t}(u)}$. Since $K_{t}$ is compact and $\bar{D}_{t}$ is continuous, we conclude that $D_{t}$ is uniformly continuous, and we can therefore find a positive real number $\rho_{t}>0$ such that if $\left(u^{\prime}, v^{\prime}\right),\left(u^{\prime \prime}, v^{\prime \prime}\right) \in K_{t}$ and $\left|\left(u^{\prime}, v^{\prime}\right)-\left(u^{\prime \prime}, v^{\prime \prime}\right)\right| \leq \rho_{t}$, then $\left|D_{t}\left(u^{\prime}, v^{\prime}\right)-D_{t}\left(u^{\prime \prime}, v^{\prime \prime}\right)\right| \leq \varepsilon$. In particular, this implies that if $(u, v) \in K_{t}$ and $v \leq \rho_{t}$, then $|(u, v)-(u, 0)|=v \leq \rho_{t}$, and so $\left|D_{t}(u, v)-D_{t}(u, 0)\right| \leq \varepsilon$, whence

$$
D_{t}(u, v) \leq D_{t}(u, 0)+\varepsilon=1+\varepsilon .
$$

Definition of $\delta_{t}$. Since $h$ is continuous, and therefore uniformly continuous on compact subintervals of $(0, \infty)$, we can find a positive real number $\delta_{t}>0$, such that if $s^{\prime}, s^{\prime \prime} \in\left[t_{0}, T_{t}\right]$ with $\left|s^{\prime}-s^{\prime \prime}\right| \leq \delta_{t}$, then

$$
\left|h\left(2 e^{-s^{\prime}}\right)-h\left(2 e^{-s^{\prime \prime}}\right)\right| \leq \frac{\varepsilon}{M_{e^{-\left(T_{t}+1\right)}}(E)} .
$$

Definition of $u_{t}$. We can clearly choose a positive number $0<u_{t}<1$ such that

$$
\left|\log u_{t}\right| \leq \min \left(1, \rho_{t}, \delta_{t}, \frac{\varepsilon}{\left(\sup _{t_{0} \leq s \leq T_{t}+1} f_{E}^{h}(s)\right)\left(\sup _{t_{0} \leq s \leq T_{t}} \pi_{t}(s)\right)}\right) .
$$


After having defined the numbers $\rho_{t}, \delta_{t}$ and $u_{t}$, we put $v_{t}=\left|\log u_{t}\right|$ and note that $u_{t} e^{-s}=e^{-\left(s+v_{t}\right)}$ for all real numbers $s$. Also, if $s \in\left[t_{0}, T_{t}\right]$, then we conclude from this and Lemma 4.3 that

$$
\begin{aligned}
f_{\bar{E}}^{h}(s) & =M_{e^{-s}}(\bar{E}) h\left(2 e^{-s}\right) \\
& \leq M_{u_{t} e^{-s}}(E) h\left(2 e^{-s}\right) \\
& =M_{e^{-\left(s+v_{t}\right.}}(E) h\left(2 e^{-s}\right) .
\end{aligned}
$$

However, since $\left|\left(s+v_{t}\right)-s\right|=v_{t}=\left|\log u_{t}\right| \leq \delta_{t}$, it follows from (4.5) that $h\left(2 e^{-s}\right) \leq$ $h\left(2 e^{-\left(s+v_{t}\right)}\right)+\frac{\varepsilon}{M_{e^{-\left(T_{t}+1\right)}(E)}}$, and (4.7) therefore implies that

$$
\begin{aligned}
f \frac{h}{E}(s) & \leq M_{e^{-\left(s+v_{t}\right)}}(E) h\left(2 e^{-s}\right) \\
& \leq M_{e^{-\left(s+v_{t}\right)}}(E)\left(h\left(2 e^{-\left(s+v_{t}\right)}\right)+\frac{\varepsilon}{M_{e^{-\left(T_{t}+1\right)}}(E)}\right) \\
& =f_{E}^{h}\left(s+v_{t}\right)+\frac{M_{e^{-\left(s+v_{t}\right)}}(E)}{M_{e^{-\left(T_{t}+1\right)}}(E)} \varepsilon .
\end{aligned}
$$

Next, note that $e^{-\left(T_{t}+1\right)} \leq e^{-\left(s+v_{t}\right)}$ (because $s \leq T_{t}$ and $v_{t}=\left|\log u_{t}\right| \leq 1$ ), whence $M_{e^{-\left(s+v_{t}\right)}}(E) \leq M_{e^{-\left(T_{t}+1\right)}}(E)$, and so $\frac{M_{e^{-\left(s+v_{t}\right.}}(E)}{M_{e^{-\left(T_{t}+1\right)}}(E)} \leq 1$. It follows from this and (4.8) that

$$
f_{\bar{E}}^{h}(s) \leq f_{E}^{h}\left(s+v_{t}\right)+\varepsilon
$$

for all $s \in\left[t_{0}, T_{t}\right]$.

Write $\varepsilon_{t}=\varepsilon \int d \Pi_{t}$ and note that it follows from (4.9) that

$$
\begin{aligned}
\int f \frac{h}{E} d \Pi_{t}= & \int_{t_{0}}^{T_{t}} f_{\bar{E}}^{h}(s) \pi_{t}(s) d s \\
\leq & \int_{t_{0}}^{T_{t}}\left(f_{E}^{h}\left(s+v_{t}\right)+\varepsilon\right) \pi_{t}(s) d s \\
= & \int_{t_{0}}^{T_{t}} f_{E}^{h}\left(s+v_{t}\right) \pi_{t}(s) d s+\varepsilon_{t} \\
= & \int_{t_{0}+v_{t}}^{T_{t}+v_{t}} f_{E}^{h}(u) \pi_{t}\left(u-v_{t}\right) d u+\varepsilon_{t} \\
= & \int_{t_{0}+v_{t}}^{T_{t}} f_{E}^{h}(u) \pi_{t}\left(u-v_{t}\right) d u+\int_{T_{t}}^{T_{t}+v_{t}} f_{E}^{h}(u) \pi_{t}\left(u-v_{t}\right) d u+\varepsilon_{t} \\
\leq & \int_{t_{0}+\eta_{t}}^{T_{t}} f_{E}^{h}(u) \pi_{t}(u) D_{t}\left(u, v_{t}\right) d u \\
& +v_{t}\left(\sup _{t_{0} \leq s \leq T_{t}+1} f_{E}^{h}(s)\right)\left(\sup _{t_{0} \leq s \leq T_{t}} \pi_{t}(s)\right)+\varepsilon_{t} .
\end{aligned}
$$

Next, it follows from the definition of $v_{t}=\left|\log u_{t}\right|$ and (4.6) that $v_{t}\left(\sup _{t_{0} \leq s \leq T_{t}+1} f_{E}^{h}(s)\right)$ $\left(\sup _{t_{0} \leq s \leq T_{t}} \pi_{t}(s)\right) \leq \varepsilon$, and we therefore conclude from (4.10) that

$$
\int f_{\bar{E}}^{h} d \Pi_{t} \leq \int_{t_{0}+v_{t}}^{T_{t}} f_{E}^{h}(u) \pi_{t}(u) D_{t}\left(u, v_{t}\right) d u+\varepsilon+\varepsilon_{t} .
$$


Also, note that for all $u \in\left[t_{0}+v_{t}, T_{t}\right]$, we have $\left(u, v_{t}\right) \in K_{t}$ and $v_{t}=\left|\log u_{t}\right| \leq \rho_{t}$ (by (4.6)), and it therefore follows from (4.4) that $D_{t}\left(u, v_{t}\right) \leq 1+\varepsilon$. We deduce from this and (4.11) that

$$
\begin{aligned}
\int f_{\bar{E}}^{h} d \Pi_{t} & \leq(1+\varepsilon) \int_{t_{0}+v_{t}}^{T_{t}} f_{E}^{h}(u) \pi_{t}(u) d u+\varepsilon+\varepsilon_{t} \\
& \leq(1+\varepsilon) \int f_{E}^{h} d \Pi_{t}+\varepsilon+\varepsilon_{t} .
\end{aligned}
$$

Since the consistency condition implies that $\varepsilon_{t}=\varepsilon \int d \Pi_{t} \rightarrow \varepsilon$ as $t \rightarrow \infty$, we conclude from (4.12) and the definitions of $\overline{\mathcal{U}}_{\Pi}^{h}(\bar{E})=\liminf _{t} \int f \frac{h}{E} d \Pi_{t}$ and $\overline{\mathcal{U}}_{\Pi}^{h}(E)=$ $\liminf _{t} \int f_{E}^{h} d \Pi_{t}$ that $\overline{\mathcal{U}}_{\Pi}^{h}(\bar{E}) \leq(1+\varepsilon) \overline{\mathcal{U}}_{\Pi}^{h}(E)+2 \varepsilon$. Finally, letting $\varepsilon \searrow 0$, gives $\overline{\mathcal{U}}_{\Pi}^{h}(\bar{E}) \leq \overline{\mathcal{U}}_{\Pi}^{h}(E)$. Similarly, using (4.12) we also conclude that $\overline{\mathcal{V}}_{\Pi}^{h}(\bar{E}) \leq \overline{\mathcal{V}}_{\Pi}^{h}(E)$.

\section{Proof of Theorem 2.5.(1)}

The purpose of this section is to prove Theorem 2.5.(1). Recall, that if $h$ is a dimension function and $X \in K_{\mathrm{GH}}$, then we define the function $f_{X}^{h}:(0, \infty) \rightarrow(0, \infty)$ by

$$
f_{X}^{h}(t)=M_{e^{-t}}(X) h\left(2 e^{-t}\right) .
$$

For a dimension function $h$, an averaging system $\Pi=\left(\Pi_{t}\right)_{t \geq t_{0}}$, and $t, c>0$, write

$$
L_{t, c}^{h, \Pi}=\left\{X \in K_{\mathrm{GH}} \mid \int f_{X}^{h} d \Pi_{t}<c\right\} .
$$

Lemma 5.1 Let $h$ be a dimension function and let $\Pi=\left(\Pi_{t}\right)_{t \geq t_{0}}$ be an averaging system. Fix $t, c>0$. Then the set $L_{t, c}^{h, \Pi}$ is open in $K_{G H}$.

Proof Write

$$
F=K_{\mathrm{GH}} \backslash L_{t, c}^{h, \Pi}=\left\{X \in K_{\mathrm{GH}} \mid \int f_{X}^{h} d \Pi_{t} \geq c\right\} .
$$

We must now prove that $F$ is closed in $K_{\mathrm{GH}}$. In order to show this, we fix a sequence $\left(X_{n}\right)_{n}$ in $F$ and $X \in K_{\mathrm{GH}}$ with $X_{n} \rightarrow X$. We must now prove that $X \in F$, i.e. we must prove that $\int f_{X}^{h} d \Pi_{t} \geq c$. We prove this inequality below. For brevity define functions $\varphi, \varphi_{n}:\left[t_{0}, \infty\right) \rightarrow[0, \infty)$ by

$$
\varphi(s)=M_{e^{-s}}(X) h\left(2 e^{-s}\right), \quad \varphi_{n}(s)=M_{e^{-s}}\left(X_{n}\right) h\left(2 e^{-s}\right) .
$$

We now prove the following three claims.

Claim 1 We have $\int \sup _{n} \varphi_{n} d \Pi_{t}<\infty$.

Proof of Claim 1 The measure $\Pi_{t}$ has compact support, and we can therefore find $T_{0} \geq t_{0}$, such that supp $\Pi_{t} \subseteq\left[t_{0}, T_{0}\right]$. It follows from Lemma 4.1 that $M_{e^{-T_{0}}}$ is upper semi-continuous, and so $\lim \sup _{n} M_{e^{-T_{0}}}\left(X_{n}\right) \leq M_{e^{-T_{0}}}(X)$. In particular, this implies that there is a constant $K$ such that $M_{e^{-T_{0}}}\left(X_{n}\right) \leq K$ for all $n$. For positive integers $n$ and $s \in\left[t_{0}, T_{0}\right]$ we therefore conclude that $\varphi_{n}(s)=M_{e^{-s}}\left(X_{n}\right) h\left(2 e^{-s}\right) \leq M_{e^{-T_{0}}}\left(X_{n}\right) h\left(2 e^{-t_{0}}\right) \leq K h\left(2 e^{-t_{0}}\right)$. Finally, since supp $\Pi_{t} \subseteq\left[t_{0}, T_{0}\right]$, it therefore follows that $\int \sup _{n} \varphi_{n} d \Pi_{t}=\int_{t_{0}}^{T_{0}} \sup _{n} \varphi_{n} d \Pi_{t} \leq$ $K h\left(2 e^{-t_{0}}\right) \Pi_{t}\left(\left[t_{0}, T_{0}\right]\right)<\infty$ This completes the proof of Claim 1. 
Claim 2 We have $c \leq \int \lim \sup _{n} \varphi_{n} d \Pi_{t}$.

Proof of Claim 2 Since $X_{n} \in F$, we conclude that $c \leq \int f_{X_{n}}^{h} d \Pi_{t}=\int \varphi_{n} d \Pi_{t}$ for all $n$, whence

$$
c \leq \limsup _{n} \int \varphi_{n} d \Pi_{t}
$$

It also follows from Claim 1 and the Reverse Fatou Lemma (see, for example, [21, Theorem 3.2.3]) that

$$
\underset{n}{\lim \sup } \int \varphi_{n} d \Pi_{t} \leq \int \lim \sup _{n} \varphi_{n} d \Pi_{t} .
$$

The desired result follows immediately from (5.2) and (5.3). This completes the proof of Claim 2.

Claim 3 For all $s \geq t_{0}$, we have $\lim \sup _{n} \varphi_{n}(s) \leq \varphi(s)$, and so $\int \lim \sup _{n} \varphi_{n} d \Pi_{t} \leq \int \varphi d \Pi_{t}$.

Proof of Claim 3 This follows from the fact that $M_{r}: K_{\mathrm{GH}} \rightarrow \mathbb{R}$ is upper semi-continuous for all $r>0$ by Lemma 4.1. This completes the proof of Claim 3.

Finally, we deduce immediately from Claims 2 and 3 that $c \leq \int \lim \sup _{n} \varphi_{n} d \Pi_{t} \leq$ $\int \varphi d \Pi_{t}=\int f_{X}^{h} d \Pi_{t}$.

Proposition 5.2 Let $h$ be a dimension function and let $\Pi=\left(\Pi_{t}\right)_{t \geq t_{0}}$ be an averaging system.

(1) For $c \in \mathbb{R}^{+}$, write

$$
T_{c}=\left\{X \in K_{G H} \mid \overline{\mathcal{U}}_{\Pi}^{h}(X) \leq c\right\}
$$

Then $T_{c}$ is co-meagre.

(2) Write

$$
T=\left\{X \in K_{G H} \mid \overline{\mathcal{U}}_{\Pi}^{h}(X)=0\right\}
$$

Then $T$ is co-meagre.

Proof (1) It suffices to show that there is a countable family $\left(G_{u}\right)_{u \in \mathbb{Q}^{+}}$of open and dense subsets $G_{u}$ of $K_{\mathrm{GH}}$ with $\cap_{u \in \mathbb{Q}^{+}} G_{u} \subseteq T_{c}$. For $u \in \mathbb{Q}^{+}$, we define the set $G_{u}$ by

$$
G_{u}=\bigcup_{u<t} L_{t, c}^{h, \Pi}
$$

recall that the set $L_{t, c}^{h, \Pi}$ is defined in (5.1). Below we prove that the sets $G_{u}$ are open and dense subsets of $K_{\mathrm{GH}}$ with $\cap_{u \in \mathbb{Q}^{+}} G_{u} \subseteq T_{c}$; this is the contents of the three claims below.

Claim $1 G_{u}$ is open in $K_{\mathrm{GH}}$.

Proof of Claim 1 This follows immediately from Lemma 5.1. This completes the proof of Claim 1.

Claim $2 G_{u}$ is dense in $K_{\mathrm{GH}}$. 
Proof of Claim 2 We first prove that $\left\{X \in K_{\mathrm{GH}} \mid X\right.$ is finite $\} \subseteq G_{u}$. Indeed, if $X$ is a finite metric space, then it is clear that $f_{X}^{h}(t)=M_{e^{-t}}(X) h\left(2 e^{-t}\right) \rightarrow 0$, and the consistency condition therefore implies that $\int f_{X}^{h} d \Pi_{t} \rightarrow 0$. It follows from this that there is a number $t$ with $t>u$ and $\int f_{X}^{h} d \Pi_{t}<c$, whence $X \in L_{t, c}^{h, \Pi} \subseteq G_{u}$. This proves that $\left\{X \in K_{\mathrm{GH}} \mid X\right.$ is finite $\} \subseteq G_{u}$.

Next, since $\left\{X \in K_{\mathrm{GH}} \mid X\right.$ is finite $\} \subseteq G_{u}$ and $\left\{X \in K_{\mathrm{GH}} \mid X\right.$ is finite $\}$ is dense in $K_{\mathrm{GH}}$, we conclude that $G_{u}$ is dense in $K_{\mathrm{GH}}$. This completes the proof of Claim 2.

Claim $3 \cap_{u \in \mathbb{Q}^{+}} G_{u} \subseteq T_{c}$.

Proof of Claim 3 Let $X \in \cap_{u \in \mathbb{Q}^{+}} G_{u}$. We must now show that $\overline{\mathcal{U}}_{\Pi}^{h}(X) \leq c$. Since $X \in$ $\cap_{u \in \mathbb{Q}^{+}} G_{u} \subseteq \cap_{n} G_{n}$, we conclude that for each positive integer $n$, we can find a positive number $t_{n}$ with $t_{n}>n$ such that $X \in L_{t_{n}, c}^{h}$, whence $\int f_{X}^{h} d \Pi_{t_{n}}<c$. It follows immediately from this that $\overline{\mathcal{U}}_{\Pi}^{h}(X)=\liminf _{t} \int f_{X}^{h} d \Pi_{t} \leq \liminf _{n} \int f_{X}^{h} d \Pi_{t_{n}} \leq c$, and so $X \in T_{c}$. This completes the proof of Claim 3.

(2) This statement follows immediately from Part (1) since $T=\cap_{c \in \mathbb{Q}^{+}} T_{c}$.

We can now prove Theorem 2.5.(1).

Proof of Theorem 2.5.(1) Theorem 2.5.(1) follows immediately from Proposition 5.2.(2).

\section{Proof of Theorem 2.5.(2)-(3)}

The purpose of this section is to prove Theorem 2.5.(2)-(3). We start by introducing the following notation. First, recall that for a positive real number $r$, the covering number $N_{r}(X)$ of a metric space $X$ is defined in (2.1). Next, for a dimension function $h$ and a metric space $X$, define the function $g_{X}^{h}:(0, \infty) \rightarrow(0, \infty)$ by

$$
g_{X}^{h}(t)=N_{e^{-t}}(X) h\left(2 e^{-t}\right) .
$$

Finally, for a dimension function $h$, an averaging system $\Pi=\left(\Pi_{t}\right)_{t \geq t_{0}}$ and $r, t, c>0$, write

$$
\Lambda_{t, c}^{h, \Pi}=\left\{X \in K_{\mathrm{GH}} \mid \int g_{X}^{h} d \Pi_{t}>c\right\},
$$

and

$$
\begin{aligned}
L_{r, t, c}^{h, \Pi}= & \left\{X \in K_{\mathrm{GH}} \mid \text { there is a positive integer } N\right. \text { and } \\
& x_{1}, \ldots, x_{N} \in X, \quad C_{1}, \ldots, C_{N} \subseteq X, t_{1}, \ldots, t_{N}>t, \\
& \text { such that } \\
& X=\cup_{i} B\left(x_{i}, r\right), \\
& C_{i} \subseteq B\left(x_{i}, r\right) \text { for all } i, \\
& \left.C_{i} \in \Lambda_{t_{i}, c}^{h, \Pi} \text { for all } i\right\} .
\end{aligned}
$$

Lemma 6.1 Let $h$ be a dimension function and let $\Pi=\left(\Pi_{t}\right)_{t \geq t_{0}}$ be an averaging system. Fix $t, c>0$. Then the set $\Lambda_{t, c}^{h, \Pi}$ is open in $K_{G H}$.

Proof Write

$$
F=K_{\mathrm{GH}} \backslash \Lambda_{t, c}^{h, \Pi}=\left\{X \in K_{\mathrm{GH}} \mid \int g_{X}^{h} d \Pi_{t} \leq c\right\} .
$$


We must now prove that $F$ is closed in $K_{\mathrm{GH}}$. In order to show this, we fix a sequence $\left(X_{n}\right)_{n}$ in $F$ and $X \in K_{\mathrm{GH}}$ with $X_{n} \rightarrow X$. We must now prove that $X \in F$, i.e. we must prove that $\int g_{X}^{h} d \Pi_{t} \leq c$. We prove this inequality below. For brevity define functions $\varphi, \varphi_{n}:\left[t_{0}, \infty\right) \rightarrow[0, \infty)$ by

$$
\varphi(s)=N_{e^{-s}}(X) h\left(2 e^{-s}\right), \quad \varphi_{n}(s)=N_{e^{-s}}\left(X_{n}\right) h\left(2 e^{-s}\right) .
$$

We now prove the following two claims.

Claim 1 We have $\int \liminf _{n} \varphi_{n} d \Pi_{t} \leq c$.

Proof of Claim 1 Since $X_{n} \in F$, we conclude that $\int \varphi_{n} d \Pi_{t}=\int g_{X_{n}}^{h} d \Pi_{t} \leq c$ for all $n$, whence $\liminf _{n} \int \varphi_{n} d \Pi_{t} \leq c$. It follows immediately from this and Fatou's Lemma that $\int \lim \inf _{n} \varphi_{n} d \Pi_{t} \leq \liminf _{n} \int \varphi_{n} d \Pi_{t} \leq c$. This completes the proof of Claim 1.

Claim 2 For all $s \geq t_{0}$, we have $\varphi(s) \leq \liminf _{n} \varphi_{n}(s)$, and so $\int \varphi d \Pi_{t} \leq \int \liminf _{n} \varphi_{n} d \Pi_{t}$.

Proof of Claim 3 This follows from the fact that $N_{r}: K_{\mathrm{GH}} \rightarrow \mathbb{R}$ is lower semi-continuous for all $r>0$ by Lemma 4.1. This completes the proof of Claim 2 .

Finally, we deduce from Claim 1 and Claim 2 that $\int g_{X}^{h} d \Pi_{t}=\int \varphi d \Pi_{t} \leq$ $\int \lim \inf _{n} \varphi_{n} d \Pi_{t} \leq c$.

Proposition 6.2 Let $h$ be a dimension function and let $\Pi=\left(\Pi_{t}\right)_{t \geq t_{0}}$ be an averaging system. Fix $r, t, c>0$. Then the set $L_{r, t, c}^{h, \Pi}$ is open in $K_{G H}$.

Proof Let $X \in L_{r, t, c}^{h, \Pi}$ and let $d_{X}$ denote the metric in $X$. We must now find $\rho>0$ such that $B(X, \rho) \subseteq L_{r, t, c}^{h}$.

Since $X \in L_{r, t, c}^{h, \Pi}$, we conclude that here is a positive integer $N$ and

$$
x_{1}, \ldots, x_{N} \in X, \quad C_{1}, \ldots, C_{N} \subseteq X, \quad t_{1}, \ldots, t_{N}>t,
$$

such that

$$
\begin{aligned}
& X=\cup_{i} B_{X}\left(x_{i}, r\right), \\
& C_{i} \subseteq B_{X}\left(x_{i}, r\right) \text { for all } i, \\
& C_{i} \in \Lambda_{t_{i}, c}^{h, \Pi} \text { for all } i .
\end{aligned}
$$

Define $\Phi: X \rightarrow \mathbb{R}$ by $\Phi(x)=\min _{i} d_{X}\left(x, x_{i}\right)$ and note that $\Phi$ is continuous. Since $X$ is compact, we conclude that there is a point $x_{0} \in X$ such that $\Phi\left(x_{0}\right)=\sup _{x \in X} \Phi(x)$. For brevity write $r_{0}=\Phi\left(x_{0}\right)=\sup _{x \in X} \Phi(x)$, and note that since $x_{0} \in X=\cup_{i} B\left(x_{i}, r\right)$, we can find $i_{0}$ with $x_{0} \in B\left(x_{i_{0}}, r\right)$, whence

$$
r_{0}=\Phi\left(x_{0}\right) \leq d_{X}\left(x, x_{i_{0}}\right)<r .
$$

Also, since $C_{i}$ is compact and $C_{i} \subseteq B\left(x_{i}, r\right)$, we conclude that

$$
r_{i}=\inf \left\{s \mid C_{i} \subseteq B\left(x_{i}, s\right)\right\}<r .
$$

For brevity write

$$
d_{i}=r-r_{i} .
$$


Finally, since $C_{i} \in \Lambda_{t_{i}, c}^{h, \Pi}$ and $\Lambda_{t_{i}, c}^{h, \Pi}$ is open (by Lemma 6.1), we conclude that there is a positive real number $\rho_{i}>0$ with

$$
B\left(C_{i}, \rho_{i}\right) \subseteq \Lambda_{t_{i}, c}^{h, \Pi} .
$$

Now put

$$
\rho=\min \left(\frac{\rho_{1}}{2}, \ldots, \frac{\rho_{N}}{2}, \frac{r-r_{0}}{2}, \frac{d_{1}}{16}, \ldots, \frac{d_{N}}{16}\right) .
$$

It follows from (6.4) and (6.5) that $\rho>0$. We will now prove that

$$
B(X, \rho) \subseteq L_{r, t, c}^{h, \Pi} .
$$

We therefore fix $Y \in B(X, \rho)$ and proceed to show that $Y \in L_{r, t, c}^{h, \Pi}$. Let $d_{Y}$ denote the metric in $Y$. Since $d_{\mathrm{GH}}(X, Y)<\rho$, it follows that we may assume that there is a complete metric space $\left(Z, d_{Z}\right)$ with $X, Y \subseteq Z$ and $d_{\mathrm{H}}(X, Y)<\rho$ such that $d_{X}\left(x^{\prime}, x^{\prime \prime}\right)=d_{Z}\left(x^{\prime}, x^{\prime \prime}\right)$ for all $x^{\prime}, x^{\prime \prime} \in X$, and $d_{Y}\left(y^{\prime}, y^{\prime \prime}\right)=d_{Z}\left(y^{\prime}, y^{\prime \prime}\right)$ for all $y^{\prime}, y^{\prime \prime} \in Y$. Below we use the following notation allowing us to distinguish balls in $Y$ and balls in $Z$. Namely, we will denote the open ball in $Y$ with radius equal to $\delta$ and centre at $y \in Y$ by $B_{Y}(y, \delta)$, i.e. $B_{Y}(y, \delta)=\left\{y^{\prime} \in Y \mid d_{Y}\left(y, y^{\prime}\right)<\delta\right\}$, and we will denote the open ball in $Z$ with radius equal to $\delta$ and centre at $z \in Z$ by $B_{Z}(z, \delta)$, i.e. $B_{Z}(z, \delta)=\left\{z^{\prime} \in X \mid d_{X}\left(z, z^{\prime}\right)<\delta\right\}$. We must now show that $Y \in L_{r, t, c}^{h, \Pi}$. Since $d_{\mathrm{H}}(X, Y)<\rho$, we conclude that for each $i$, there is a point $y_{i} \in Y$ with $d_{Z}\left(x_{i}, y_{i}\right)<\rho$. Next, put

$$
K_{i}=\left\{y \in Y \mid \operatorname{dist}\left(y, C_{i}\right) \leq \rho\right\} .
$$

It is clear that

$$
y_{1}, \ldots, y_{N} \in Y, \quad K_{1}, \ldots, K_{N} \subseteq Y, \quad t_{1}, \ldots, t_{N}>t,
$$

Hence, to prove that $Y \in L_{r, t, c}^{h, \Pi}$, it suffices to show that

$$
\begin{aligned}
& Y=\cup_{i} B_{Y}\left(y_{i}, r\right), \\
& K_{i} \subseteq B_{Y}\left(y_{i}, r\right) \text { for all } i, \\
& K_{i} \in \Lambda_{t_{i}, c}^{h, \Pi} \text { for all } i .
\end{aligned}
$$

The proofs of (6.8)-(6.10) are the contents of the three claims below.

Claim $1 Y=\cup_{i} B_{Y}\left(y_{i}, r\right)$.

Proof of Claim 1 It is clear that $\cup_{i} B_{Y}\left(y_{i}, r\right) \subseteq Y$. In order to prove the reverse inclusion, we let $y \in Y$. Since $d_{\mathrm{H}}(X, Y)<\rho$, we conclude that there is a point $x \in X$ with $d_{Z}(x, y)<\rho$. Also, since $\min _{i} d_{X}\left(x, x_{i}\right)=\Phi(x) \leq r_{0}$, we deduce that there is an index $j$ with $d_{X}\left(x, x_{j}\right) \leq$ $r_{0}$. Finally, it follows from the definition of $y_{j}$ that $d_{Z}\left(x_{j}, y_{j}\right)<\rho$. Hence $d_{Y}\left(y, y_{j}\right)=$ $d_{Z}\left(y, y_{j}\right) \leq d_{Z}(y, x)+d_{Z}\left(x, x_{j}\right)+d_{Z}\left(x_{j}, y_{j}\right)=d_{Z}(y, x)+d_{X}\left(x, x_{j}\right)+d_{Z}\left(x_{j}, y_{j}\right)<$ $\rho+r_{0}+\rho=2 \rho+r_{0} \leq r$, and so $y \in B_{Y}\left(y_{j}, r\right) \subseteq \cup_{i} B_{Y}\left(y_{i}, r\right)$. This completes the proof of Claim 1.

Claim $2 K_{i} \subseteq B\left(y_{i}, r\right)$ for all $i$.

Proof of Claim 2 Since $C_{i} \subseteq B_{X}\left(x_{i}, r\right)$, it follows from the definition of the numbers $r_{i}=$ $\inf \left\{s \mid C_{i} \subseteq B\left(x_{i} s\right)\right\}$ and $d_{i}=r-r_{i}$, that

$$
C_{i} \subseteq B_{X}\left(x_{i}, r-\frac{d_{i}}{2}\right) .
$$


Next, since $d_{Z}\left(x_{i}, y_{i}\right)<\rho \leq \frac{d_{i}}{16} \leq \frac{d_{i}}{4}$, it follows that

$$
\begin{aligned}
B_{X}\left(x_{i}, r-\frac{d_{i}}{2}\right) & \subseteq B_{Z}\left(x_{i}, r-\frac{d_{i}}{2}\right) \\
& \subseteq B_{Z}\left(y_{i}, r-\frac{d_{i}}{4}\right) .
\end{aligned}
$$

Finally, combining (6.11) and (6.12) shows that

$$
C_{i} \subseteq B_{Z}\left(y_{i}, r-\frac{d_{i}}{4}\right) .
$$

We can now prove that $K_{i} \subseteq B_{Y}\left(y_{i}, r\right)$. Let $y \in K_{i}$. Since $y \in K_{i}$, we have $\operatorname{dist}_{Z}\left(y, C_{i}\right) \leq$ $\rho \leq \frac{d_{i}}{16}<\frac{d_{i}}{8}$, and it therefore follows that there is $x \in C_{i}$ with $d_{Z}(x, y) \leq \frac{d_{i}}{8}$. Also, we deduce from (6.13) that $x \in C_{i} \subseteq B_{Z}\left(y_{i}, r-\frac{d_{i}}{4}\right)$, whence $d_{Z}\left(x, y_{i}\right) \leq r-\frac{d_{i}}{4}$. Combining the previous inequalities we have $d_{Y}\left(y, y_{i}\right)=d_{Z}\left(y, y_{i}\right) \leq d_{Z}(y, x)+d_{Z}\left(x, y_{i}\right) \leq \frac{d_{i}}{8}+r-\frac{d_{i}}{4}<$ $r$, and so $y \in B_{Y}\left(y_{i}, r\right)$. This completes the proof of Claim 2 .

Claim $3 K_{i} \in \Lambda_{t_{i}, c}^{h, \Pi}$ for all $i$.

Proof of Claim 3 It is clear that $K_{i}$ is a closed subset of $Y$ and so $K_{i} \in K_{\mathrm{GH}}$.

We now prove that

$$
\sup _{x \in C_{i}} \operatorname{dist}_{Z}\left(x, K_{i}\right) \leq \rho .
$$

Indeed, let $x \in C_{i}$. Since $d_{\mathrm{H}}(X, Y)<\rho$, we conclude that there is $y \in Y$ such that $d_{Z}(x, y)<$ $\rho$. In particular, since $x \in C_{i}$, this shows that $\operatorname{dist}\left(y, C_{i}\right) \leq d_{Z}(y, x) \leq \rho$, and so $y \in K_{i}$. We deduce from this that $\operatorname{dist}\left(x, K_{i}\right) \leq d_{Z}(x, y) \leq \rho$. Finally, taking supremum over all $x \in C_{i}$ shows that $\sup _{x \in C_{i}} \operatorname{dist}\left(x, K_{i}\right) \leq \rho$. This completes the proof of (6.14).

Next, we prove that

$$
\sup _{y \in K_{i}} \operatorname{dist}\left(y, C_{i}\right) \leq \rho .
$$

Indeed, let $y \in K_{i}$. Since $y \in K_{i}$, it follows from the definition of $K_{i}$ that there is $x \in C_{i}$ such that $d_{Z}(y, x) \leq \rho$, and so $\operatorname{dist}\left(y, C_{i}\right) \leq d_{Z}(y, x) \leq \rho$. Finally, taking supremum over all $y \in K_{i}$ shows that $\sup _{y \in D_{i}} \operatorname{dist}\left(y, C_{i}\right) \leq \rho$. This completes the proof of (6.15).

Combining (6.14) and (6.15), we immediately conclude that $d_{\mathrm{H}}\left(C_{i}, K_{i}\right)=\max \left(\sup _{x \in C_{i}}\right.$ $\left.\operatorname{dist}\left(x, K_{i}\right), \sup _{y \in K_{i}} \operatorname{dist}\left(y, C_{i}\right)\right) \leq \rho \leq \frac{\rho_{i}}{2}<\rho_{i}$, whence $K_{i} \in B\left(C_{i}, \rho_{i}\right) \subseteq \Lambda_{t_{i}, c}^{h, \Pi}$. This completes the proof of Claim 3.

It follows immediately from Claims $1-3$ that $Y \in L_{r, t, c}^{h}$.

Proposition 6.3 Let $h$ be a continuous dimension function and let $\Pi=\left(\Pi_{t}\right)_{t \geq t_{0}}$ be an averaging system.

(1) For $c \in \mathbb{R}^{+}$, write

$$
T_{c}=\left\{X \in K_{G H} \mid \overline{\mathcal{V}}_{\Pi}^{h}(U) \geq c \text { for all open subsets } U \text { of } X \text { with } U \neq \varnothing\right\} .
$$

Then $T_{c}$ is co-meagre.

(2) Write

$$
T=\left\{X \in K_{G H} \mid \overline{\mathcal{V}}_{\Pi}^{h}(U)=\infty \text { for all open subsets } U \text { of } X \text { with } U \neq \varnothing\right\} .
$$

Then $T$ is co-meagre. 
(3) Write

$$
S=\left\{X \in K_{G H} \mid \mathcal{V}_{\Pi}^{h}(U)=\infty \text { for all open subsets } U \text { of } X \text { with } U \neq \varnothing\right\} .
$$

If, in addition, $\overline{\mathcal{V}}_{\Pi}^{h}(\bar{E})=\overline{\mathcal{V}}_{\Pi}^{h}(E)$ for all metric spaces and all $E \subseteq X$, then $S$ is comeagre.

Proof (1) It suffices to show that there is a countable family $\left(G_{r, u}\right)_{r, u \in \mathbb{Q}^{+}}$of open and dense subsets $G_{r, u}$ of $K_{\mathrm{GH}}$ such that $\cap_{r, u \in \mathbb{Q}^{+}} G_{r, u} \subseteq T_{c}$. For $r, u \in \mathbb{Q}^{+}$, we define the set $G_{r, u}$ by

$$
G_{r, u}=\bigcup_{u<t} L_{r, t, c}^{h, \Pi}
$$

recall, that the set $L_{r, t, c}^{h, \Pi}$ is defined in (6.2)-(6.3). We now prove that the sets $G_{r, u}$ are open and dense subsets of $K_{\mathrm{GH}}$ such that $\cap_{r, u \in \mathbb{Q}^{+}} G_{r, u} \subseteq T_{c}$; this is the contents of the three claims below.

Claim $1 G_{r, u}$ is open in $K_{\mathrm{GH}}$.

Proof of Claim 1 This follows immediately from Proposition 6.2. This completes the proof of Claim 1.

Claim $2 G_{r, u}$ is dense in $K_{\mathrm{GH}}$.

Proof of Claim 2 Let $X \in K_{\mathrm{GH}}$ and $\rho>0$. Also, let $d_{X}$ denote the metric in $X$. We must now find $Y \in G_{r, u}$ such that $d_{\mathrm{GH}}(X, Y)<\rho$.

Firstly, since $X$ is compact, we can find a finite subset $E$ of $X$ such that

$$
d_{\mathrm{H}}(X, E)<\frac{\rho}{2} .
$$

Note that it is clear that we can find a constant $k>0$ such that for all $n \in \mathbb{N}$, all $s>0$ and all $\delta>0$, we have

$$
N_{\delta}\left(\left\{z \in \mathbb{R}^{n}|| z \mid \leq s\right\}\right) \geq k \delta^{-n} .
$$

Next, choose real numbers $T, t$ such that $T>t>u$, and for positive integers $n$, define $\varphi_{n}:\left(t_{0}, \infty\right) \rightarrow(0, \infty)$ by

$$
\varphi_{n}(s)=e^{s n} h\left(2 e^{-s}\right) .
$$

Note that $\varphi_{n} \nearrow \infty$, and it therefore follows from the Monotone Convergence theorem that $\int \varphi_{n} d \Pi_{T} \nearrow \int \infty d \Pi_{T}=\infty$. In particular, this implies that we can choose a positive integer $N$ with

$$
\int \varphi_{N} d \Pi_{T} \geq \frac{c}{k}
$$

Put $\delta_{0}=\min \left(\frac{\rho}{2}, r\right)$ and let $C=\left\{z \in \mathbb{R}^{N}|| z \mid \leq \delta_{0}\right\}$. Finally, we define the space $Y$ by

$$
Y=E \times C
$$

and equip $Y$ with the supremum metric $d_{Y}$ induced by $d_{X}$ and $|\cdot|$, i.e. $d_{Y}\left(\left(x^{\prime}, z^{\prime}\right),\left(x^{\prime \prime}, z^{\prime \prime}\right)\right)=$ $\max \left(d_{X}\left(x^{\prime}, x^{\prime \prime}\right),\left|z^{\prime}-z^{\prime \prime}\right|\right)$ for $x^{\prime}, x^{\prime \prime} \in E$ and $z^{\prime}, z^{\prime \prime} \in C$. It is clear that $Y$ is compact, and so $Y \in K_{\mathrm{GH}}$. Below we show that $d_{\mathrm{GH}}(X, Y)<\rho$ and $Y \in G_{r, u}$. 
Proof of $d_{\mathrm{GH}}(X, Y)<\rho$. Define $f: E \rightarrow Y$ and $g: Y \rightarrow Y$ by $f(x)=(x, 0)$ and $g: Y \rightarrow Y$ by $g(x, z)=(x, z)$. It is clear that $f$ and $g$ are isometries and we therefore conclude that $\left.d_{\mathrm{GH}}(E, Y) \leq d_{\mathrm{H}}(f(E), g(Y))=d_{\mathrm{H}}(E \times\{0\}\}, E \times C\right) \leq \sup _{z \in C}|z|=\delta_{0} \leq \frac{\rho}{2}$, whence $d_{\mathrm{GH}}(X, Y) \leq d_{\mathrm{GH}}(X, E)+d_{\mathrm{GH}}(E, Y)<d_{\mathrm{H}}(X, E)+\frac{\rho}{2}<\frac{\rho}{2}+\frac{\rho}{2}=\rho$. This completes the proof of $d_{\mathrm{GH}}(X, Y)<\rho$.

Proof of $Y \in G_{r, u}$. Write $E=\left\{x_{1}, \ldots, x_{M}\right\}$ and put

$$
y_{i}=\left(x_{i}, 0\right), \quad C_{i}=\left\{x_{i}\right\} \times C, \quad t_{i}=T,
$$

for $i=1, \ldots, M$. In order to prove that $Y \in G_{r, u}$, it suffices the show that $Y \in L_{r, t, c}^{h, \Pi}$, and in order to show that $Y \in L_{r, t, c}^{h, \Pi}$, it clearly suffices to prove that

$$
\begin{aligned}
Y & =\cup_{i} B\left(y_{i}, r\right), \\
C_{i} & \subseteq B\left(y_{i}, r\right) \text { for all } i, \\
C_{i} & =\Lambda_{t_{i}, c}^{h, \Pi} \text { for all } i .
\end{aligned}
$$

Below we show that the statements in (6.18)-(6.20) are satisfied.

Indeed, it is clear that $Y=E \times C=\cup_{i}\left(\left\{x_{i}\right\} \times C\right)=\cup_{i} B\left(y_{i}, r\right)$; this proves (6.18).

It is also clear that $C_{i} \subseteq B\left(y_{i}, r\right)$ for all $i$; this proves (6.19).

Finally, we prove (6.20). We have

$$
\begin{aligned}
\int g_{C_{i}}^{h} d \Pi_{t_{i}} & =\int N_{e^{-s}}\left(C_{i}\right) h\left(2 e^{-s}\right) d \Pi_{T}(s) \\
& =\int N_{e^{-s}}\left(\left\{x_{i}\right\} \times C\right) h\left(2 e^{-s}\right) d \Pi_{T}(s) \\
& =\int N_{e^{-s}}(C) h\left(2 e^{-s}\right) d \Pi_{T}(s) .
\end{aligned}
$$

However, it follows from (6.16) that $N_{e^{-s}}(C) \geq k e^{s N}$, and we therefore conclude from (6.21) that

$$
\int g_{C_{i}}^{h} d \Pi_{t_{i}} \geq k \int e^{s N} h\left(2 e^{-s}\right) d \Pi_{T}(s)=k \int \varphi_{N} d \Pi_{T} .
$$

Finally, combining (6.17) and (6.22), we now deduce that $\int g_{C_{i}}^{h} d \Pi_{t_{i}} \geq k \frac{c}{k}=c$, whence $C_{i} \in \Lambda_{t_{i}, c}^{h, \Pi}$. This completes the proof of (6.20).

It follows immediately from (6.18)-(6.20) that $Y \in L_{r, t, c}^{h, \Pi} \subseteq G_{r, u}$. This completes the proof of Claim 2.

Claim $3 \cap_{r, u \in \mathbb{Q}^{+}} G_{r, u} \subseteq T_{c}$.

Proof of Claim 3 Let $X \in \cap_{r, u \in \mathbb{Q}^{+}} G_{r, u}$. We must now show that if $U$ is an open subset of $X$ with $U \neq \varnothing$, then $\overline{\mathcal{V}}_{\Pi}^{h}(U) \geq c$. We therefore let $U$ be an open subset of $X$ with $U \neq \varnothing$, and proceed to show that $\overline{\mathcal{V}}_{\Pi}^{h}(U) \geq c$. Since $U$ is non-empty and open there is $x_{0} \in U$ and $r_{0}>0$ with $B\left(x_{0}, r_{0}\right) \subseteq U$. Next, since $X \in \cap_{r, u \in \mathbb{Q}^{+}} G_{r, u} \subseteq \cap_{n} G_{\frac{r_{0}}{2}, n}$, we conclude that for each positive integer $n$, we can find a positive real number $t_{n}$ with $t_{n}>n$ such that $X \in L_{\frac{r_{0}}{2}, t_{n}, c}^{h, \Pi}$. In particular, this implies that there is a positive integer $N_{n}$ and

$$
x_{n, 1}, \ldots, x_{n, N_{n}} \in X, \quad C_{n, 1}, \ldots, C_{n, N_{n}} \subseteq X, \quad t_{n, 1}, \ldots, t_{n, N_{n}}>t_{n},
$$


such that

$$
\begin{aligned}
& X=\cup_{i} B\left(x_{n, i}, \frac{r_{0}}{2}\right), \\
& C_{n, i} \subseteq B\left(x_{n, i}, \frac{r_{0}}{2}\right) \text { for all } i, \\
& C_{n, i} \in \Lambda_{t_{n, i}, c}^{h, \Pi} \text { for all } i .
\end{aligned}
$$

Since $x_{0} \in X=\cup_{i} B\left(x_{n, i}, \frac{r_{0}}{2}\right)$, we can choose an index $i_{n} \in\left\{1, \ldots, N_{n}\right\}$ such that $x_{0} \in B\left(x_{n, i_{n}}, \frac{r_{0}}{2}\right)$, whence $B\left(x_{n, i_{n}}, \frac{r_{0}}{2}\right) \subseteq B\left(x_{0}, r_{0}\right)$, and so $C_{n, i_{n}} \subseteq B\left(x_{n, i_{n}}, \frac{r_{0}}{2}\right) \subseteq$ $B\left(x_{0}, r_{0}\right) \subseteq U$. Since it follows from Lemma 4.1 that $f_{U}^{h}(s) \geq g_{U}^{h}(s)$ for all $s$, we conclude from this combined with the fact that $C_{n, i_{n}} \in \Lambda_{t_{n, i_{n}, c}}^{h, \Pi}$, that $\int f_{U}^{h} d \Pi_{t_{n, i_{n}}} \geq \int g_{U}^{h} d \Pi_{t_{n, i_{n}}} \geq$ $\int g_{C_{n, i_{n}}}^{h} d \Pi_{t_{n, i_{n}}} \geq c$. Finally, since $t_{n, i_{n}}>t_{n}>n$ and so $t_{n, i_{n}} \rightarrow \infty$, we deduce from the previous inequality that $\overline{\mathcal{V}}_{\Pi}^{h}(U)=\limsup _{t} \int f_{U}^{h} d \Pi_{t} \geq \lim \sup _{n} \int f_{U}^{h} d \Pi_{t_{n, i_{n}}} \geq c$. This completes the proof of Claim 3.

(2) This statement follows immediately from Part (1) since clearly $T=\cap_{c \in \mathbb{Q}^{+}} T_{c}$.

(3) Using Part (2), it clearly suffices to prove that

$$
T \subseteq S .
$$

We will now prove (6.23). Let $X \in T$. We must now show that $X \in S$, i.e. we must show that if $U$ is an open subset of $X$ with $U \neq \varnothing$, then $\mathcal{V}_{\Pi}^{h}(U)=\infty$. We therefore let $U$ be an open subset of $X$ with $U \neq \varnothing$, and proceed to show that $\mathcal{V}_{\Pi}^{h}(U)=\infty$. Since $U$ is non-empty and open there is $x \in U$ and $r>0$ such that $B_{X}(x, r) \subseteq U$. In particular, if we write $C=\overline{B\left(x, \frac{r}{2}\right)}$, then $C$ is compact and $C \subseteq B(x, r) \subseteq U$. Next, we prove the following claim.

Claim 4 If $V$ is an open subset of $X$ with $V \cap C \neq \varnothing$, then $\overline{\mathcal{V}}_{\Pi}^{h}(V \cap C)=\infty$.

Proof of Claim 4 Let $V$ be an open subset of $X$ with $V \cap C \neq \varnothing$. We must now show that $\overline{\mathcal{V}}_{\Pi}^{h}(V \cap C)=\infty$. As $V \cap C \neq \varnothing$, it is possible to choose $y \in V \cap C$. Since $y \in V$ and $V$ is open, we can choose $\varepsilon>0$ such that $B(y, \varepsilon) \subseteq V$. Next, since $y \in C=\overline{B\left(x, \frac{r}{2}\right)}$, we choose $z \in B\left(x, \frac{r}{2}\right)$ such that $z \in B(y, \varepsilon)$. Finally, since $z \in B\left(x, \frac{r}{2}\right) \cap B(y, \varepsilon)$, we can find $\delta>0$ with $B(z, \delta) \subseteq B\left(x, \frac{r}{2}\right) \cap B(y, \varepsilon)$, whence $B(z, \delta) \subseteq B\left(x, \frac{r}{2}\right) \cap B(y, \varepsilon) \subseteq C \cap V$, and so

$$
\overline{\mathcal{V}}_{\Pi}^{h}(B(z, \delta)) \leq \overline{\mathcal{V}}_{\Pi}^{h}(C \cap V) .
$$

However, since $B(z, \delta)$ is open and non-empty and $X \in T$, it follows that $\overline{\mathcal{V}}_{\Pi}^{h}(B(z, \delta))=\infty$, and we therefore conclude from (6.24) that $\overline{\mathcal{V}}_{\Pi}^{h}(C \cap V)=\infty$. This completes the proof of Claim 4.

Finally, it follows immediately from Claim 4 and Lemma 4.2 that $\mathcal{V}_{\Pi}^{h}(C)=\infty$, and since $C \subseteq U$, this implies that $\mathcal{V}_{\Pi}^{h}(U)=\infty$.

We can now prove Theorem 2.5.(2)-(3).

Proof of Theorem 2.5.(2)-(3) The statements in Theorems 2.5.(2) and 2.5.(3) follow immediately from the statements in Propositions 6.3.(2) and 6.3.(3), respectively.

Open Access This article is distributed under the terms of the Creative Commons Attribution 4.0 International License (http://creativecommons.org/licenses/by/4.0/), which permits unrestricted use, distribution, and reproduction in any medium, provided you give appropriate credit to the original author(s) and the source, provide a link to the Creative Commons license, and indicate if changes were made. 


\section{References}

1. Balka, R., Máthé, A.: Generalized Hausdorff measure for generic compact sets. Ann. Acad. Sci. Fenn. Math. 38, 797-804 (2013)

2. Edgar, G.: Integral, Probability, and Fractal Measures. Springer, New York (1998)

3. Falconer, K.J.: Fractal Geometry. Wiley, Chichester (1989)

4. Feng, D.-J., Wu, J.: Category and dimension of compact subsets of $\mathbb{R}^{n}$. Chin. Sci. Bull. 42, 1680-1683 (1997)

5. Gruber, P.: Dimension and structure of typical compact sets, continua and curves. Monatsh. Math. 108, 149-164 (1989)

6. Gruber, P., Zamfirescu, T.: Generic properties of compact starshaped sets. Proc. AMS 108, 207-214 (1990)

7. Haase, H.: A contribution to measure and dimension of metric spaces. Math. Nachr. 124, 45-55 (1985)

8. Haase, H.: Open-invariant measures and the covering number of sets. Math. Nachr. 134, 295-307 (1987)

9. Hardy, G.: Divergent Series. Oxford University Press, Oxford (1949)

10. Hewitt, E., Stromberg, K.: Real and Abstract Analysis. A Modern Treatment of the Theory of Functions of a Real Variable. Springer, New York (1965)

11. Jacob, M.: Über die Äquivalenz der Cesaroschen und der Hölderschen Mittel für Integrale bei gleicher reeller Ordnung $k>0$. Math. Z. 26, 672-682 (1927)

12. Jurina, S., MacGregor, N., Mitchell, A., Olsen, L., Stylianou, A.: On the Hausdorff and packing measures of typical compact metric spaces. Aequ. Math. 92, 709-735 (2018)

13. Mattila, P.: Geometry of Sets and Measures in Euclidean Spaces, Fractals and Rectifiability, Cambridge Studies in Advanced Mathematics, vol. 44. Cambridge University Press, Cambridge (1995)

14. Munroe, M.: Introduction to Measure and Integration. Addison-Wesley, Cambridge (1953)

15. Myjak, J., Rudnicki, R.: Box and packing dimensions of typical compact sets. Monatsh. Math. 131, 223-226 (2000)

16. Oxtoby, J.: Measure and Category. A Survey of the Analogies Between Topological and Measure Spaces, Graduate Texts in Mathematics, vol. 2. Springer, New York (1971)

17. Pesin, Y.: Dimension Theory in Dynamical Systems, Contemporary views and applications, Chicago Lectures in Mathematics. University of Chicago Press, Chicago (1997)

18. Petersen, P.: Riemannian Geometry, 2nd edn. Graduate Texts in Mathematics, vol. 171. Springer, New York (2006)

19. Rogers, C.A.: Hausdorff Measures. Cambridge University Press, London (1970)

20. Rouyer, J.: Generic properties of compact metric spaces. Topol. Appl. 158, 2140-2147 (2011)

21. Stroock, D.: Essentials of Integration Theory for Analysis. Springer, New York (2011)

22. Zindulka, O.: Packing measures and dimensions on Cartesian products. Publ. Mat. 57, 393-420 (2013)

Publisher's Note Springer Nature remains neutral with regard to jurisdictional claims in published maps and institutional affiliations. 